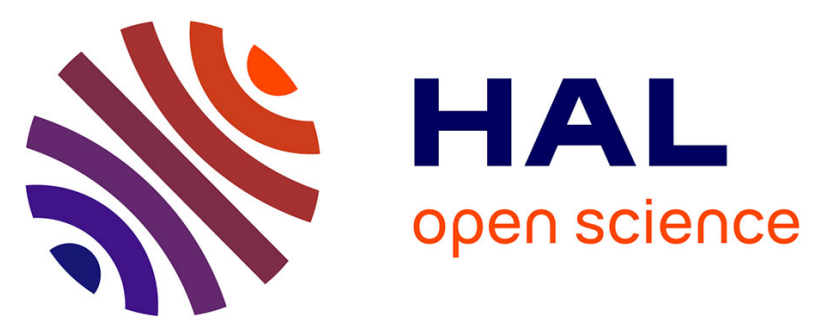

\title{
A FULLY WELL-BALANCED, POSITIVE AND ENTROPY-SATISFYING GODUNOV-TYPE METHOD FOR THE SHALLOW-WATER EQUATIONS
}

\author{
Christophe Berthon, Christophe Chalons
}

\section{To cite this version:}

Christophe Berthon, Christophe Chalons. A FULLY WELL-BALANCED, POSITIVE AND ENTROPY-SATISFYING GODUNOV-TYPE METHOD FOR THE SHALLOW-WATER EQUA-

TIONS. Mathematics of Computation, 2016, 85 (299), pp.1281-1307. hal-00956799v2

HAL Id: hal-00956799

https://hal.science/hal-00956799v2

Submitted on 30 Apr 2015

HAL is a multi-disciplinary open access archive for the deposit and dissemination of scientific research documents, whether they are published or not. The documents may come from teaching and research institutions in France or abroad, or from public or private research centers.
L'archive ouverte pluridisciplinaire HAL, est destinée au dépôt et à la diffusion de documents scientifiques de niveau recherche, publiés ou non, émanant des établissements d'enseignement et de recherche français ou étrangers, des laboratoires publics ou privés. 


\title{
A FULLY WELL-BALANCED, POSITIVE AND \\ ENTROPY-SATISFYING GODUNOV-TYPE METHOD FOR THE SHALLOW-WATER EQUATIONS
}

\author{
CHRISTOPHE BERTHON* AND CHRISTOPHE CHALONS ${ }^{\dagger}$
}

AMS subject classifications. 65M60, 65M12, 76M12, 35L65

Key words. Shallow-water equations, steady states, finite volume schemes, well-balanced property, positive preserving scheme, entropy preserving scheme

Abstract. This work is devoted to the derivation of a fully well-balanced numerical scheme for the well-known shallow-water model. During the last two decades, several well-balanced strategies have been introduced with a special attention to the exact capture of the stationary states associated with the so-called lake at rest. By fully well-balanced, we mean here that the proposed Godunov-type method is also able to preserve stationary states with non zero velocity. The numerical procedure is shown to preserve the positiveness of the water height and satisfies a discrete entropy inequality.

1. Introduction. The present work is devoted to the derivation of numerical schemes to approximate the solutions of the well-known shallow-water model

$$
\left\{\begin{array}{l}
\partial_{t} h+\partial_{x}(h u)=0 \\
\partial_{t}(h u)+\partial_{x}\left(h u^{2}+g \frac{h^{2}}{2}\right)=-g h \partial_{x} z
\end{array}\right.
$$

where $z: \mathbb{R} \rightarrow \mathbb{R}$ denotes a given smooth topography and $g>0$ stands for the gravity constant. This model governs the evolution of the positive water depth and the depth-average velocity; namely $h$ and $u$.

To shorten the notations, we rewrite (1.1) as follows:

$$
\partial_{t} \mathbf{w}+\partial_{x} \mathbf{f}(\mathbf{w})=\mathbf{s}(\mathbf{w}, z),
$$

with $\mathbf{w}=\left(\begin{array}{c}h \\ h u\end{array}\right), \quad \mathbf{f}(\mathbf{w})=\left(\begin{array}{c}h u \\ h u^{2}+g \frac{h^{2}}{2}\end{array}\right), \quad \mathbf{s}(\mathbf{w}, z)=\left(\begin{array}{c}0 \\ -g h \partial_{x} z\end{array}\right)$. We here denote $\Omega$ the natural phase space defined by $\Omega=\left\{\mathbf{w}=(h, h u)^{T} \in \mathbb{R}^{2} ; h>0\right\}$. From now on, let us emphasize we do not consider dry areas where $h=0$. The reader is referred for instance to $[1,6,5,30]$ where the wet/dry transition regions are of prime interest and suitable numerical techniques to deal with such transitions can be found therein the cited references.

In fact, the present work focuses on the steady states governed by

$$
\left\{\begin{array}{l}
\partial_{x}(h u)=0 \\
\partial_{x}\left(h u^{2}+g \frac{h^{2}}{2}\right)=-g h \partial_{x} z
\end{array}\right.
$$

\footnotetext{
*Université de Nantes, Laboratoire de Mathématiques Jean Leray, CNRS UMR 6629, 2 rue de la Houssinière, BP 92208, 44322 Nantes, France.

${ }^{\dagger}$ Laboratoire de Mathématiques de Versailles, UMR 8100, Université de Versailles Saint-Quentinen-Yvelines, UFR des Sciences, bâtiment Fermat, 45 avenue des Etats-Unis, 78035 Versailles cedex, France.
} 
Therefore, the smooth steady states under consideration are given by

$$
\left\{\begin{array}{l}
h u=\mathrm{cst} \\
\frac{u^{2}}{2}+g(h+z)=\mathrm{cst} .
\end{array}\right.
$$

Among this full familly of steady states, the lake at rest

$$
h+z=\mathrm{cst}, \quad u=0,
$$

certainly is of main importance. During the last two decades, after the works by Bermudez-Vasquez [2] and Greenberg-LeRoux [24] (see also [21, 22, 23]), the derivation of well-balanced schemes able to restore the lake at rest (1.4) was a very active research topic. Several strategies have been derived (for instance see $[1,30,26,10,21,24,11$, $32,31,34,33,12,38,39])$. The main difficulty coming from the derivation of wellbalanced schemes remains in the discretization of the topography source term to be consistent with the lake at rest. In [1], a well-balanced strategy was introduced, the so-called hydrostatic reconstruction, to enforce the source term discretization to be consistent with the lake at rest independently from the finite volume scheme involved to approximate the homogeneous system issuing from (1.1). In this brief state of the art devoted to well-balanced schemes for the shallow-water equations, let us mention a recent improvement of the hydrostatic reconstruction as the generalized hydrostatic reconstruction [13] or the upwind hydrostatic approach [5].

In fact, all the above cited references (except for [13]) deal only with the lake at rest steady states (1.4) but they do not consider the steady states (1.3) in their full generality. As a consequence, a natural question arises. Does there exist a numerical strategy able to restore all the steady states (1.3)? Such strategies exist and, up to our knowledge, a few attempts can be found in [13], [9] and [40] (see also [41], [42] and [43]). In [13], the main idea stays in a suitable extension of the hydrostatic reconstruction. The resulting scheme is able to restore all the steady states (1.3) but the scheme fails preserving the positiveness of the water depth. Next, in [9] the authors argue a suitable relaxation model to design a numerical scheme able to preserve the subsonic $(|u|<\sqrt{g h})$ steady states. This relaxation approach does not preserve all the steady states, but a large familly and it ensures the water depth to be positive. In addition, this relaxation scheme is proved to be stable since it satisfies discrete entropy inequalities. Indeed, recall that the system (1.1) is endowed with the following entropy inequality related to the physical energy:

$$
\begin{aligned}
& \partial_{t} \mathcal{U}(\mathbf{w})+\partial_{x} \mathcal{F}(\mathbf{w}) \leq-g h u \partial_{x} z, \\
& \mathcal{U}(\mathbf{w})=h \frac{u^{2}}{2}+g \frac{h^{2}}{2}, \quad \mathcal{F}(\mathbf{w})=\left(\frac{u^{2}}{2}+g h\right) h u,
\end{aligned}
$$

which rewrites for smooth solutions $\partial_{t}(\mathcal{U}(\mathbf{w})+g h z)+\partial_{x}(\mathcal{F}(\mathbf{w})+g h z u) \leq 0$. The derivation of entropy preserving schemes turns out to be, in general, a delicate problem. The reader is referred, for instance, to $[6,3,14,15,7,8]$ where entropy stable schemes are developped for the shallow-water model or related systems. In [40], the author develops a well-balanced discontinuous Galerkin method which preserves not only the still water at rest steady state, but also the more general moving water equilibrium. The key idea is a special source term approximation coupled with an approximation of the numerical fluxes based on a generalized hydrostatic reconstruction. This paper is more focused on high-order approximations thanks to the DG approach, 
but does not address the entropy inequality property. Unlike the present paper which is only first-order accurate but can consider the entropy inequality property quite easily thanks to the use of a suitable approximate Riemann solver. Note also the related papers [41], [42] and [43] which show the advantage of the full well-balanced property over classical well-balanced schemes by means of several numerical examples.

In the present work, we exhibit a Godunov-type scheme [25] which is:

- water depth positivity preserving,

- entropy preserving,

- fully well-balanced (able to restore all the steady states).

The paper is organized as follows. For the sake of consistency of the present paper, in the next section we briefly recall the main algebraic properties satisfied by (1.1) and we emphasize that the steady states (1.3) can be understood as specific Riemann invariants of the model. In addition, we exhibit an unconsistency coming from the discontinuous steady states and we thus give a precise definition of these equilibrium states of interest. In Section 3, we propose several remarks about the derivation of approximate Riemann solvers and the associated Godunov-type schemes. In particular, we recall that the (exact) Godunov scheme obviously satisfies all the required properties but for a non-analytical solvable Riemann problem. Therefore, we introduce our strategy by considering an approximate Riemann solver made of constant states including the topography source term in a sense to be specified. In fact, the relevant introduction of the source term inside the approximate Riemann solver imposes to consider an additional nonlinear relation. Firstly, in Section 4, we propose a suitable linearization of this non-standard relation to easily obtain a fully well-balanced and positivity preserving scheme. We note that this first naive approach illustrates the relevance of our approximate Riemann solver including the source term in its definition. Indeed, at this level, we have straighforwardly designed a numerical scheme with better property than the generalized hydrostatic reconstruction. However, the stability of this first approach is not established. In order to derive an entropy preserving scheme, we suggest to consider the new nonlinear relation coming from the source term in its full generality. Then, Section 5 is devoted to the study of this relation and the characterization of the adopted approximate Riemann solver. Finally, we establish all the required properties; namely fully well-balanced property, positivity preserving and entropy preserving.

2. Basic properties. For the sake of completeness, we recall some properties satisfied by (1.1) (e.g. see $[6,19,29])$. These properties will be useful in the forthcoming developments. First, let us rewrite the model in the following equivalent form:

$$
\left\{\begin{array}{l}
\partial_{t} h+\partial_{x}(h u)=0 \\
\partial_{t}(h u)+\partial_{x}\left(h u^{2}+g \frac{h^{2}}{2}\right)+g h \partial_{x} z=0 \\
\partial_{t} z=0
\end{array}\right.
$$

To simplify the notations, we introduce the following condensed form:

$$
\partial_{t} \mathbf{v}+\partial_{x} \mathbf{f}_{z}(\mathbf{v})+\mathbf{b}(\mathbf{v}) \partial_{x} \mathbf{v}=0
$$


where we have set $\mathbf{v}=\left(\begin{array}{c}h \\ h u \\ z\end{array}\right), \mathbf{f}_{z}(\mathbf{v})=\left(\begin{array}{c}h u \\ h u^{2}+g \frac{h^{2}}{2} \\ 0\end{array}\right), \mathbf{b}(\mathbf{v})=\left(\begin{array}{ccc}0 & 0 & 0 \\ 0 & 0 & g h \\ 0 & 0 & 0\end{array}\right)$.

We denote $\Omega_{z}$ the natural phase space defined by

$$
\Omega_{z}=\left\{\mathbf{v}=(h, h u, z)^{T} \in \mathbb{R}^{3} ; h>0\right\} .
$$

Concerning the algebra of (2.1), the eigenvalues of the matrix

$$
\nabla_{\mathbf{v}} \mathbf{f}_{z}(\mathbf{v})+\mathbf{b}(\mathbf{v})=\left(\begin{array}{ccc}
0 & 1 & 0 \\
-u^{2}+g h & 2 u & g h \\
0 & 0 & 0
\end{array}\right)
$$

are given by $\lambda_{-}=u-c \leq \lambda_{0}=0 \leq \lambda_{+}=u+c$, where $c=\sqrt{g h}$ denotes the sound speed. The corresponding eigenvectors are

$$
r_{-}(\mathbf{v})=\left(\begin{array}{c}
1 \\
u-c \\
0
\end{array}\right), \quad r_{0}(\mathbf{v})=\left(\begin{array}{c}
c^{2} \\
0 \\
u^{2}-c^{2}
\end{array}\right), \quad r_{+}(\mathbf{v})=\left(\begin{array}{c}
1 \\
u+c \\
0
\end{array}\right),
$$

and form a basis of $\mathbb{R}^{3}$ provided that $u \neq \pm c$. The states $\mathbf{v} \in \Omega_{z}$ such that $u= \pm c$ are said to be sonic. Similarly, the states $\mathbf{v} \in \Omega_{z}$ such that $|u|<c$, respectively $|u|>c$, are said to be subsonic, respectively supersonic. System (2.1) is then strictly hyperbolic on the set of subsonic and supersonic states. The reader is referred to [28] where an analysis of the resonant regime $|u|=c$ is performed.

Characteristic fields and Riemann invariants. Easy calculations give

$$
\nabla_{\mathbf{v}} \lambda_{-}(\mathbf{v}) \cdot r_{-}(\mathbf{v})=-\frac{3 c}{2 h}<0, \quad \nabla_{\mathbf{v}} \lambda_{0}(\mathbf{v}) \cdot r_{0}(\mathbf{v})=0, \quad \nabla_{\mathbf{v}} \lambda_{+}(\mathbf{v}) \cdot r_{+}(\mathbf{v})=\frac{3 c}{2 h}>0,
$$

so that the characteristic fields associated with $\lambda_{-}$and $\lambda_{+}$are genuinely non linear, while the characteristic field associated with $\lambda_{0}$ is linearly degenerate, see for instance [20] for more details. In addition, the Riemann invariants $\left(I_{-}^{(l)}\right)_{l=1,2},\left(I_{0}^{(l)}\right)_{l=1,2}$ and $\left(I_{+}^{(l)}\right)_{l=1,2}$ respectively associated with $\lambda_{-}, \lambda_{0}$ and $\lambda_{+}$are given by

$$
I_{ \pm}^{(1)}=z, \quad I_{ \pm}^{(2)}=u-( \pm 2 c) \quad \text { and } \quad I_{0}^{(1)}=h u, \quad I_{0}^{(2)}=\frac{u^{2}}{2}+g(h+z) .
$$

From now on, let us underline that the Riemann invariants associated with the stationary characteristic field are nothing but the steady states (1.3).

Entropy and admissible discontinuities. Smooth solutions of (2.1) are defined in the classical sense without ambiguity. Using a chain rule argument, they satisfy the additional conservation law $\partial_{t} \tilde{\mathcal{U}}(\mathbf{v})+\partial_{x} \tilde{\mathcal{F}}(\mathbf{v})=0$, where the entropy-entropy flux pair $(\tilde{\mathcal{U}}, \tilde{\mathcal{F}})$ is given by

$$
\tilde{\mathcal{U}}(\mathbf{v})=h\left(\frac{u^{2}-g h}{2}+g(h+z)\right), \quad \tilde{\mathcal{F}}(\mathbf{v})=h u\left(\frac{u^{2}}{2}+g(h+z)\right) .
$$

The usual entropy inequality associated with (2.1) and used as a selection principle for discontinuous solutions, see again [20], then writes

$$
\partial_{t} \tilde{\mathcal{U}}(\mathbf{v})+\partial_{x} \tilde{\mathcal{F}}(\mathbf{v}) \leq 0 .
$$


As far as these discontinuous solutions are concerned, let us consider the step function

$$
\mathbf{v}(x, t)=\left\{\begin{array}{lll}
\mathbf{v}_{1} & \text { if } \quad x<\sigma t \\
\mathbf{v}_{2} & \text { if } \quad x>\sigma t
\end{array}\right.
$$

where $\mathbf{v}_{1}$ and $\mathbf{v}_{2}$ belong to $\Omega_{z}$ and $\sigma \in \mathbb{R}$ represents the speed of propagation of the discontinuity, and let us distinguish between the two cases $z_{1}=z_{2}$ and $z_{1} \neq z_{2}$. When $z_{1}=z_{2},(2.1)$ is made of two conservation laws since $\partial_{x} z=0$. The step function (2.4) is then called a shock discontinuity and is said to be admissible provided that $(2.2)$ and (2.3) are satisfied in the distributional sense, that is to say provided that the Rankine-Hugoniot relations

$$
-\sigma\left(\mathbf{v}_{2}-\mathbf{v}_{1}\right)+\mathbf{f}_{z}\left(\mathbf{v}_{2}\right)-\mathbf{f}_{z}\left(\mathbf{v}_{1}\right)=0
$$

and the entropy inequality

$$
-\sigma\left(\tilde{\mathcal{U}}\left(\mathbf{v}_{2}\right)-\tilde{\mathcal{U}}\left(\mathbf{v}_{1}\right)\right)+\left(\tilde{\mathcal{F}}\left(\mathbf{v}_{2}\right)-\tilde{\mathcal{F}}\left(\mathbf{v}_{1}\right)\right) \leq 0
$$

hold true. When $z_{1} \neq z_{2},(2.1)$ is no longer conservative so that the classical RankineHugoniot relations do not make sense anymore. However, we note that since $\partial_{t} z=0$, we necessarily have $\sigma=0$ and then $\sigma=\lambda_{0}$, which means that the discontinuity (2.4) is associated with a linearly degenerate characteristic field, see again [20]. In this context, it is classical to define the admissibility criterion by the continuity of the Riemann invariants $\left(I_{0}^{(l)}\right)_{l=1,2}$. In other words, $(2.4)$ is said to be admissible if

$$
\left\{\begin{array}{l}
I_{0}^{(1)}\left(\mathbf{v}_{1}\right)=I_{0}^{(1)}\left(\mathbf{v}_{2}\right) \\
I_{0}^{(2)}\left(\mathbf{v}_{1}\right)=I_{0}^{(2)}\left(\mathbf{v}_{2}\right) .
\end{array}\right.
$$

In this case, note that we have by definition of $I_{0}^{(1)}$ and $I_{0}^{(2)}$ the validity of the following entropy equality:

$$
-\sigma\left(\tilde{\mathcal{U}}\left(\mathbf{v}_{2}\right)-\tilde{\mathcal{U}}\left(\mathbf{v}_{1}\right)\right)+\left(\tilde{\mathcal{F}}\left(\mathbf{v}_{2}\right)-\tilde{\mathcal{F}}\left(\mathbf{v}_{1}\right)\right)=\left(\tilde{\mathcal{F}}\left(\mathbf{v}_{2}\right)-\tilde{\mathcal{F}}\left(\mathbf{v}_{1}\right)\right)=0 .
$$

The step function (2.4) is then called a contact discontinuity.

To conclude this section, it is crucial to emphasize that, because of the resonant regime, a shock wave given by (2.5)-(2.6) may coincide with a stationary wave defined by (2.7). As a consequence, we have $\sigma=0$ within $z_{1}=z_{2}$. In this case, on one hand the Rankine-Hugoniot relations and the entropy inequality respectively write

$$
\left\{\begin{array}{l}
q:=h_{1} u_{1}=h_{2} u_{2}, \\
h_{1} u_{1}^{2}+g \frac{h_{1}^{2}}{2}=h_{2} u_{2}^{2}+g \frac{h_{2}^{2}}{2},
\end{array} \quad \text { and } \quad q\left(\left(\frac{u_{2}^{2}}{2}+g h_{2}\right)-\left(\frac{u_{1}^{2}}{2}+g h_{1}\right)\right) \leq 0 .\right.
$$

On the other hand, (2.7) governs the stationary wave and write here

$$
\left\{\begin{array}{l}
q:=h_{1} u_{1}=h_{2} u_{2} \\
\frac{u_{1}^{2}}{2}+g h_{1}=\frac{u_{2}^{2}}{2}+g h_{2}
\end{array}\right.
$$

It is worth noticing that (2.9) and (2.10) cannot be satisfied simultaneously except for $u_{1}=u_{2}=0$. In other words, the admissibility criteria suffer from a lack of continuity 
at point $z_{1}=z_{2}$ for stationary discontinuities. As expected, this shortcoming will be also observed when designing an approximate Riemann solver for (1.1).

Since the steady states are of primary importance in the present work, to avoid such an unconsistency, we suggest to impose the steady states to be smooth. Therefore, in the sequel, we will consider the following definition.

Assumption 2.1. The steady states for the shallow-water model (1.1) are smooth functions $\mathbf{w}: \mathbb{R} \rightarrow \Omega$ such that (1.3) holds.

Since we have imposed a smooth topography, in the present work we deal with smooth steady states. The reader is referred to $[13,33,38,39]$ where extension to discontinuous buttom is considered that makes discontinuous the steady water height (see also $[28,29]$ to related studies).

3. Godunov-type schemes. Let us briefly recall the definition of the Godunovtype scheme. We first introduce a space step $\Delta x$ and a time step $\Delta t$, both assumed to be constant for simplicity in the forthcoming developments. We set $\nu=\Delta t / \Delta x$. We define the mesh interfaces $x_{j+1 / 2}=j \Delta x$ for $j \in \mathbb{Z}$ and the intermediate times $t^{n}=n \Delta t$ for $n \in \mathbb{N}$. Then at each time $t^{n}$ and on the control cell $\left[x_{j-1 / 2}, x_{j+1 / 2}\right)$, $j \in \mathbb{Z}$, we assume known an approximation $\mathbf{w}_{j}^{n}$ of the solution $\mathbf{w}$ of (1.1) with a given initial data $x \mapsto \mathbf{w}_{0}(x)$. Therefore, a piecewise constant approximate solution $x \mapsto \mathbf{w}_{\nu}^{n}\left(x, t^{n}\right)$ of the solution $\mathbf{w}$ is given by

$$
\mathbf{w}_{\nu}^{n}\left(x, t^{n}\right)=\mathbf{w}_{j}^{n} \text { for all } x \in\left[x_{j-1 / 2} ; x_{j+1 / 2}\right), \quad j \in \mathbb{Z}, n \in \mathbb{N} .
$$

As usual [20], the approximation of the inital data is given by

$$
\mathbf{w}_{j}^{0}=\frac{1}{\Delta x} \int_{x_{j-1 / 2}}^{x_{j+1 / 2}} \mathbf{w}_{0}(x) d x .
$$

Given a smooth function $x \rightarrow z(x)$, we also define $z_{j}=\frac{1}{\Delta x} \int_{x_{j-1 / 2}}^{x_{j+1 / 2}} z(x) d x$. We now make $\left(\mathbf{w}_{i}^{n}\right)_{i \in \mathbb{Z}}$ evolve to the next time level $t^{n+1}$ by considering a Godunov-type scheme. These approaches are composed of two steps: a first step is devoted to make the initial data evolve in time according to the model under consideration, and a second step is concerned with a projection onto piecewise constant functions.

Step 1 : Evolution in time

In this first step, one approximates the solution of (1.1) with initial data $x \mapsto \mathbf{w}_{\nu}^{n}\left(x, t^{n}\right)$ and for times $t \in[0, \Delta t]$ with $\Delta t$ small enough. This approximate solution, denoted $\mathbf{w}_{\nu}^{n+1}\left(x, t^{n}+t\right)$, is known by gluing together approximated solutions of the Riemann problems set at each interface. More precisely

$$
\mathbf{w}_{\nu}^{n+1}\left(x, t^{n}+t\right)=\mathbf{w}_{\mathcal{R}}\left(\frac{x-x_{j+1 / 2}}{t-t^{n}} ; \mathbf{v}_{j}^{n}, \mathbf{v}_{j+1}^{n}\right) \text { for all }(x, t) \in\left[x_{j}, x_{j+1}\right] \times[0, \Delta t],
$$

where $\mathbf{w}_{\mathcal{R}}\left(x / t ; v_{L}, v_{R}\right)$ stands for a suitable approximation of the solution of the Riemann problem associated with (1.1), or equivalently with (2.2), with initial

$$
\mathbf{w}_{0}(x):=\mathbf{w}(x, 0)=\left\{\begin{array}{ll}
\mathbf{w}_{L} & \text { if } x<0, \\
\mathbf{w}_{R} & \text { if } x>0,
\end{array} \quad \text { and } z(x)=\left\{\begin{array}{lll}
z_{L} & \text { if } \quad x<0 \\
z_{R} & \text { if } \quad x>0
\end{array}\right.\right.
$$

where $\mathbf{v}_{L}=\left(\mathbf{w}_{L}, z_{L}\right)$ and $\mathbf{v}_{R}=\left(\mathbf{w}_{R}, z_{R}\right)$ belong to the phase space $\Omega_{z}$. Let us underline the dependence on $\mathbf{v}_{L}$ and $\mathbf{v}_{R}$ inside $\mathbf{w}_{\mathcal{R}}$ instead of $\mathbf{w}_{L}$ and $\mathbf{w}_{R}$, to highlight 
the dependence on the topography $z$.

Let us now define an extended definition of a steady state solution in agreement with Definition 2.1, which will be useful in what follows.

DEFINITION 3.1. The states $\mathbf{v}_{L}$ and $\mathbf{v}_{R}$ define a steady state solution if and only if the following relations are satisfied:

$$
\begin{aligned}
& \left\|\mathbf{v}_{R}-\mathbf{v}_{L}\right\|=\mathcal{O}(\Delta x) \quad \text { and } \quad\left(u_{L}^{2}-c_{L}^{2}\right)\left(u_{R}^{2}-c_{R}^{2}\right)>0, \\
& h_{L} u_{L}=h_{R} u_{R}, \\
& \frac{u_{L}^{2}}{2}+g\left(h_{L}+z_{L}\right)=\frac{u_{R}^{2}}{2}+g\left(h_{R}+z_{R}\right) .
\end{aligned}
$$

Note that the natural condition $\left(u_{L}^{2}-c_{L}^{2}\right)\left(u_{R}^{2}-c_{R}^{2}\right)>0$ imposes that both states are either subsonic or supersonic (see for instance [16]).

Step 2 : Projection $\left(t^{n} \rightarrow t^{n+1}\right)$

Aim of this second step is to get a piecewise constant approximate solution on each cell $\left[x_{j-1 / 2}, x_{j+1 / 2}\right)$ at time $t^{n+1}$. This may be simply done by averaging the solution $x \mapsto \mathbf{w}_{\nu}^{n+1}\left(x, t^{n}+\Delta t\right)$ given by (3.1), as expressed by the following update formula :

$$
\mathbf{w}_{j}^{n+1}=\frac{1}{\Delta x} \int_{x_{j-1 / 2}}^{x_{j+1 / 2}} \mathbf{w}_{\nu}^{n+1}\left(x, t^{n}+\Delta t\right) d t, j \in \mathbb{Z} .
$$

We have now to propose a relevant defintion of $\mathbf{w}_{\mathcal{R}}$ in order to derive schemes able to satisfy all the required properties. Before we detail the proposed approximate Riemann solvers, we give the properties to be satisfied by the function $\mathbf{w}_{\mathcal{R}}$ in order to obtain a relevant numerical scheme.

THEOREM 3.2. For all $j$ in $\mathbb{Z}$, assume $\mathbf{w}_{j}^{n}$ in $\Omega$ and let $\mathbf{v}_{L}$ and $\mathbf{v}_{R}$ belong to $\Omega_{z}$.

(i) Assume the following integral consistency condition:

$$
\begin{aligned}
& \frac{1}{\Delta x} \int_{-\Delta x / 2}^{\Delta x / 2} \mathbf{w}_{\mathcal{R}}\left(\frac{x}{\Delta t} ; \mathbf{v}_{L}, \mathbf{v}_{R}\right) d x= \\
& \quad \frac{1}{2}\left(\mathbf{w}_{L}+\mathbf{w}_{R}\right)-\frac{\Delta t}{\Delta x}\left(\mathbf{f}\left(\mathbf{w}_{R}\right)-\mathbf{f}\left(\mathbf{w}_{L}\right)\right)+\Delta t \overline{\mathbf{s}}\left(\Delta t, \Delta x ; \mathbf{v}_{L}, \mathbf{v}_{R}\right),
\end{aligned}
$$

where $\overline{\mathbf{s}}\left(\Delta t, \Delta x ; \mathbf{v}_{L}, \mathbf{v}_{R}\right)$ denotes an approximation of the source term in (1.1):

$$
\lim _{\substack{\mathbf{v}_{L}, \mathbf{v}_{R} \rightarrow \mathbf{v} \\ \Delta t, \Delta x \rightarrow 0}} \overline{\mathbf{s}}\left(\Delta t, \Delta x ; \mathbf{v}_{L}, \mathbf{v}_{R}\right)=\mathbf{s}(\mathbf{v}) .
$$

Then the updated formula (3.6) is consistent with (1.1) and we have

$$
\begin{gathered}
\mathbf{w}_{j}^{n+1}=\mathbf{w}_{j}^{n}-\frac{\Delta t}{\Delta x}\left(\mathbf{f}_{j+1 / 2}-\mathbf{f}_{j-1 / 2}\right)+\Delta t \mathbf{s}_{j}^{n}, \\
\mathbf{f}_{j+1 / 2}=\frac{1}{2}\left(\mathbf{f}\left(\mathbf{w}_{j}^{n}\right)+\mathbf{f}\left(\mathbf{w}_{j+1}^{n}\right)\right)-\frac{\Delta x}{4 \Delta t}\left(\mathbf{w}_{j+1}^{n}-\mathbf{w}_{j}^{n}\right)- \\
\quad \frac{1}{2 \Delta t} \int_{0}^{\Delta x / 2} \mathbf{w}_{\mathcal{R}}\left(\frac{x}{\Delta t} ; \mathbf{w}_{j}^{n}, \mathbf{w}_{j+1}^{n}\right) d x+ \\
\quad+\frac{1}{2 \Delta t} \int_{-\Delta x / 2}^{0} \mathbf{w}_{\mathcal{R}}\left(\frac{x}{\Delta t} ; \mathbf{w}_{j}^{n}, \mathbf{w}_{j+1}^{n}\right) d x, \\
\mathbf{s}_{j}^{n}=\frac{1}{2}\left(\overline{\mathbf{s}}\left(\Delta t, \Delta x ; \mathbf{v}_{j-1}^{n}, \mathbf{v}_{j}^{n}\right)+\overline{\mathbf{s}}\left(\Delta t, \Delta x ; \mathbf{v}_{j}^{n}, \mathbf{v}_{j+1}^{n}\right)\right) .
\end{gathered}
$$


(ii) For all $(x, t) \in \mathbb{R} \times \mathbb{R}_{+}$, assume $\mathbf{w}_{\mathcal{R}}\left(\frac{x}{t} ; \mathbf{v}_{L}, \mathbf{v}_{R}\right)$ belongs to $\Omega$. Then $\mathbf{w}_{j}^{n+1}$ stays in $\Omega$ for all $j \in \mathbb{Z}$.

(iii) If $\mathbf{v}_{L}$ and $\mathbf{v}_{R}$ define a steady state according to Definition 3.1, assume the following stationary property:

$$
\mathbf{w}_{\mathcal{R}}\left(\frac{x}{t} ; \mathbf{v}_{L}, \mathbf{v}_{R}\right)=\mathbf{w}_{0}(x), \quad \forall(x, t) \in \mathbb{R} \times \mathbb{R}_{+} .
$$

Then, if $\left(\mathbf{w}_{j}^{n}\right)_{j \in \mathbb{Z}}$ define a steady state, we get $\mathbf{w}_{j}^{n+1}=\mathbf{w}_{j}^{n}$ for all $j$ in $\mathbb{Z}$.

(iv) Assume the following relation holds:

$$
\begin{aligned}
& \frac{1}{\Delta x} \int_{-\Delta x / 2}^{\Delta x / 2} \mathcal{U}\left(\mathbf{w}_{\mathcal{R}}\left(\frac{x}{\Delta t} ; \mathbf{v}_{L}, \mathbf{v}_{R}\right)\right) d x \leq \\
& \frac{1}{2}\left(\mathcal{U}\left(\mathbf{w}_{L}\right)+\mathcal{U}\left(\mathbf{w}_{R}\right)\right)-\frac{\Delta t}{\Delta x}\left(\mathcal{F}\left(\mathbf{w}_{R}\right)-\mathcal{F}\left(\mathbf{w}_{L}\right)\right)+\Delta t \sigma_{\nu}\left(\Delta t, \Delta x ; \mathbf{v}_{L}, \mathbf{v}_{R}\right),
\end{aligned}
$$

where $\lim _{\mathbf{v}_{L}, \mathbf{v}_{R} \rightarrow \mathbf{v}} \sigma_{\nu}\left(\Delta t, \Delta x ; \mathbf{v}_{L}, \mathbf{v}_{R}\right)=-g h u \partial_{x} z$. Then, the numerical scheme defined by (3.6) is entropy preserving:

$$
\mathcal{U}\left(\mathbf{w}_{j}^{n+1}\right) \leq \mathcal{U}\left(\mathbf{w}_{j}^{n}\right)-\frac{\Delta t}{\Delta x}\left(\mathcal{F}_{j+1 / 2}-\mathcal{F}_{j-1 / 2}\right)+\Delta t \sigma_{j}^{n},
$$

with $\mathcal{F}_{j+1 / 2}$ and $\sigma_{j}^{n}$ defined in a similar way to the flux and source term in the update formula.

Proof. To establish (i), we follow the work by Harten et al.[25]. By definition of $\mathbf{w}_{j}^{n+1}$, given by $(3.6)$, we write

$$
\begin{aligned}
& \mathbf{w}_{j}^{n+1}= \frac{1}{\Delta x} \int_{x_{j-1 / 2}}^{x_{j}} \mathbf{w}_{\mathcal{R}}\left(\frac{x-x_{j-1 / 2}}{\Delta t} ; \mathbf{v}_{j-1}^{n}, \mathbf{v}_{j}^{n}\right) d x+ \\
& \frac{1}{\Delta x} \int_{x_{j}}^{x_{j+1 / 2}} \mathbf{w}_{\mathcal{R}}\left(\frac{x-x_{j+1 / 2}}{\Delta t} ; \mathbf{v}_{j}^{n}, \mathbf{v}_{j+1}^{n}\right) d x \\
&=\frac{\Delta t}{2 \Delta x}\left(\frac{1}{\Delta t} \int_{0}^{\Delta x / 2} \mathbf{w}_{\mathcal{R}}\left(\frac{x}{\Delta t} ; \mathbf{v}_{j-1}^{n}, \mathbf{v}_{j}^{n}\right) d x-\frac{1}{\Delta t} \int_{0}^{\Delta x / 2} \mathbf{w}_{\mathcal{R}}\left(\frac{x}{\Delta t} ; \mathbf{v}_{j}^{n}, \mathbf{v}_{j+1}^{n}\right) d x\right)+ \\
& \frac{\Delta t}{2 \Delta x}\left(-\frac{1}{\Delta t} \int_{-\Delta x / 2}^{0} \mathbf{w}_{\mathcal{R}}\left(\frac{x}{\Delta t} ; \mathbf{v}_{j-1}^{n}, \mathbf{v}_{j}^{n}\right) d x+\frac{1}{\Delta t} \int_{-\Delta x / 2}^{0} \mathbf{w}_{\mathcal{R}}\left(\frac{x}{\Delta t} ; \mathbf{v}_{j}^{n}, \mathbf{v}_{j+1}^{n}\right) d x\right)+ \\
& \frac{1}{2 \Delta x} \int_{-\Delta x / 2}^{\Delta x / 2} \mathbf{w}_{\mathcal{R}}\left(\frac{x}{\Delta t} ; \mathbf{v}_{j}^{n}, \mathbf{v}_{j+1}^{n}\right) d x+\frac{1}{2 \Delta x} \int_{-\Delta x / 2}^{\Delta x / 2} \mathbf{w}_{\mathcal{R}}\left(\frac{x}{\Delta t} ; \mathbf{v}_{j-1}^{n}, \mathbf{v}_{j}^{n}\right) d x .
\end{aligned}
$$

Involving (3.7), we immediately get the updated formula (3.9)-(3.10)-(3.11). Next, as soon as the approximate Riemann solver preserves the water depth positive, we immediately deduce the statement (ii). Similarly, concerning the property (iii), as long as $\left(\mathbf{w}_{j}^{n}\right)_{j \in \mathbb{Z}}$ defines a steady state, the relations (3.15) and (3.12) give:

$$
\mathbf{w}_{j}^{n+1}=\frac{1}{\Delta x} \int_{x_{j-1 / 2}}^{x_{j}} \mathbf{w}_{j}^{n} d x+\frac{1}{\Delta x} \int_{x_{j}}^{x_{j-1 / 2}} \mathbf{w}_{j}^{n} d x=\mathbf{w}_{j}^{n} .
$$

To prove the last property (iv), we firstly argue the Jensen inequality. Indeed, since $\mathbf{w} \mapsto \mathcal{U}(\mathbf{w})$ is convex, we have

$$
\mathcal{U}\left(\mathbf{w}_{j}^{n+1}\right) \leq \frac{1}{\Delta x} \int_{x_{j-1 / 2}}^{x_{j+1 / 2}} \mathcal{U}\left(\mathbf{w}_{\nu}^{n+1}\left(x, t^{n}+\Delta t\right)\right) d x
$$




$$
\begin{aligned}
& \leq \frac{1}{2}\left(\frac{1}{\Delta x} \int_{0}^{\Delta x / 2} \mathcal{U}\left(\mathbf{w}_{\mathcal{R}}\left(\frac{x}{\Delta t} ; \mathbf{v}_{j-1}^{n}, \mathbf{v}_{j}^{n}\right)\right) d x-\frac{1}{\Delta x} \int_{0}^{\Delta x / 2} \mathcal{U}\left(\mathbf{w}_{\mathcal{R}}\left(\frac{x}{\Delta t} ; \mathbf{v}_{j}^{n}, \mathbf{v}_{j+1}^{n}\right)\right) d x\right)+ \\
& \frac{1}{2}\left(-\frac{1}{\Delta x} \int_{-\Delta x / 2}^{0} \mathcal{U}\left(\mathbf{w}_{\mathcal{R}}\left(\frac{x}{\Delta t} ; \mathbf{v}_{j-1}^{n}, \mathbf{v}_{j}^{n}\right)\right) d x+\frac{1}{\Delta x} \int_{-\Delta x / 2}^{0} \mathcal{U}\left(\mathbf{w}_{\mathcal{R}}\left(\frac{x}{\Delta t} ; \mathbf{v}_{j}^{n}, \mathbf{v}_{j+1}^{n}\right)\right) d x\right)+ \\
& \quad \frac{1}{2 \Delta x} \int_{-\Delta x / 2}^{\Delta x / 2} \mathcal{U}\left(\mathbf{w}_{\mathcal{R}}\left(\frac{x}{\Delta t} ; \mathbf{v}_{j}^{n}, \mathbf{v}_{j+1}^{n}\right)\right) d x+\frac{1}{2 \Delta x} \int_{-\Delta x / 2}^{\Delta x / 2} \mathcal{U}\left(\mathbf{w}_{\mathcal{R}}\left(\frac{x}{\Delta t} ; \mathbf{v}_{j-1}^{n}, \mathbf{v}_{j}^{n}\right)\right) d x
\end{aligned}
$$

to get the required discrete entropy inequality (3.14). The proof is thus achieved.

3.1. The Godunov scheme. To define the function $\mathbf{w}_{\mathcal{R}}$, the most convenient choice is to consider the exact Riemann solution of (1.1) or equivalently (2.2). As it is common (see for instance [27], [20]), the self-similar Riemann solution $(x, t) \rightarrow$ $\mathbf{w}\left(x / t ; \mathbf{v}_{L}, \mathbf{v}_{R}\right)$ is made of the juxtaposition of the so-called simple waves associated with the different eigenvalues $\lambda_{ \pm}$and $\lambda_{0}$ of the model. More precisely, by simple waves we mean here the well-known rarefaction waves (smooth solutions of (2.2) depending only on $x / t$ ) and shock discontinuities associated with the genuinely non linear characteristic fields, i.e. $\lambda_{ \pm}$, and the contact disconinuities associated with the linearly degenerate characteristic field, i.e. $\lambda_{0}$. Admissible shock and contact discontinuities have been defined in the previous section.

By adopting the exact Riemann solution, let us recall that the time step $\Delta t$ is restricted to the following CFL condition:

$$
\frac{\Delta t}{\Delta x} \max _{\mathbf{v}}\left\{\left|\lambda_{ \pm}(\mathbf{v})\right|,\left|\lambda_{0}(\mathbf{v})\right|\right\} \leq \frac{1}{2}
$$

In addition, let us remark that straightforward computations give a precise definition of the approximate source term $\overline{\mathbf{s}}$ involved in (3.7):

$$
\overline{\mathbf{s}}\left(\Delta t, \Delta x ; \mathbf{v}_{L}, \mathbf{v}_{R}\right)=\frac{1}{\Delta t \Delta x} \int_{-\Delta x / 2}^{\Delta x / 2} \int_{0}^{\Delta t} \mathbf{s}\left(\mathbf{w}_{\mathcal{R}}\left(\frac{x}{t} ; \mathbf{v}_{L}, \mathbf{v}_{R}\right)\right) d x d t
$$

The main benefit of involving exact Riemann solution remains in an easy satisfaction of the required properties. Indeed, Theorem 3.2 clearly holds by definition of $\mathbf{w}_{\mathcal{R}}$.

Let us emphasize that without additional information, the Riemann solution remains unknown at this stage when $z_{L} \neq z_{R}$, at least for two reasons (see for instance $[28,19])$. The first one comes from the fact that the system $(2.7)$ defining the admissibility of the contact discontinuities may admit zero, one or two solutions $\left(h_{2}, h_{2} u_{2}\right)$ if we consider as given $\left(h_{1}, h_{1} u_{1}, z_{1}\right)$ and $z_{2}$. The second reason is related to the resonance phenomenon which means that $\lambda_{+}$or $\lambda_{-}$may equal $\lambda_{0}$ if $|u|=c$. This renders indeed the resolution of the Riemann problem even more complex. Note however that different methods can provide existence and uniqueness of an entropy solution. We refer again the reader to $[28,19]$ and the references therein.

3.2. Simple Approximate Riemann Solution and Godunov-type Scheme. To avoid the exact resolution of the Riemann solution, we now adopt some linearization approaches as proposed in $[17,18]$ (see also [14]). We briefly review the notion of simple approximate Riemann solver and the associated consistency property in the integral sense (3.7). We also derive the corresponding Godunov-type scheme and refer to $[17,18]$ for the details.

Let us consider a simple approximate Riemann solver $\mathbf{w}_{\mathcal{R}}\left(\frac{x}{t} ; \mathbf{v}_{L}, \mathbf{v}_{R}\right)$ made of $l+1$ intermediate states $\mathbf{w}_{k}$ separated by discontinuities propagating with velocities 
$\lambda_{k}=\lambda_{k}\left(\mathbf{v}_{L}, \mathbf{v}_{R}\right), k=1, \ldots, l$, namely

$$
\mathbf{w}_{\mathcal{R}}\left(\frac{x}{t} ; \mathbf{v}_{L}, \mathbf{v}_{R}\right)= \begin{cases}\mathbf{w}_{1}=\mathbf{w}_{L}, & \frac{x}{t}<\lambda_{1}, \\ \vdots & \lambda_{k-1}<\frac{x}{t}<\lambda_{k}, \\ \mathbf{w}_{k}, & \\ \vdots & \frac{x}{t}>\lambda_{l} .\end{cases}
$$

Now, the integral consistency condition (3.7) reads:

$$
\mathbf{f}\left(\mathbf{w}_{R}\right)-\mathbf{f}\left(\mathbf{w}_{L}\right)+\Delta x \overline{\mathbf{s}}\left(\Delta x, \Delta t ; \mathbf{v}_{L}, \mathbf{v}_{R}\right)=\sum_{k=1}^{l} \lambda_{k}\left(\mathbf{w}_{k+1}-\mathbf{w}_{k}\right)
$$

and where $\mathbf{s}\left(\Delta x, \Delta t ; \mathbf{v}_{L}, \mathbf{v}_{R}\right)$ is consistent with $\mathbf{s}(\mathbf{v})$ and satisfies (3.8).

By applying Theorem 3.2 (i), under the CFL condition

$$
\max _{1 \leq k \leq l}\left|\lambda_{k}\left(\mathbf{v}_{j}^{n}, \mathbf{v}_{j+1}^{n}\right)\right| \frac{\Delta t}{\Delta x} \leq \frac{1}{2}, \quad \forall j \in \mathbb{Z}
$$

we obtain the following Godunov-type scheme (see also [17, 18]):

$$
\left\{\begin{aligned}
& \mathbf{w}_{j}^{n+1}=\mathbf{w}_{j}^{n}-\frac{\Delta t}{\Delta x_{j}}\left(\mathbf{f}_{j+\frac{1}{2}}^{n}-\mathbf{f}_{j-\frac{1}{2}}^{n}\right)- \\
& \frac{\Delta t}{2 \Delta x}\left(\overline{\mathbf{s}}\left(\Delta t, \Delta x ; \mathbf{v}_{j+1}^{n}, \mathbf{v}_{j}^{n}\right)+\overline{\mathbf{s}}\left(\Delta t, \Delta x ; \mathbf{v}_{j}^{n}, \mathbf{v}_{j-1}^{n}\right)\right), \\
& \mathbf{f}_{j+\frac{1}{2}}^{n}=\mathbf{f}\left(\mathbf{w}_{j}^{n}, \mathbf{w}_{j+1}^{n}\right),
\end{aligned}\right.
$$

with $\mathbf{f}\left(\mathbf{w}_{L}, \mathbf{w}_{R}\right)=\frac{1}{2}\left\{\mathbf{f}\left(\mathbf{w}_{L}\right)+\mathbf{f}\left(\mathbf{w}_{R}\right)-\sum_{k=1}^{l}\left|\lambda_{k}\right|\left(\mathbf{w}_{k+1}-\mathbf{w}_{k}\right)\right\}$. Now, we have to design a suitable approximation of the source term, namely to propose a precise characterization of $\overline{\mathbf{s}}$, such that all the assumptions stated in Theorem 3.2 are satisfied. To adress such an issue, we adopt an approximate Riemann solver made of two intermediate states, $l=3$ in (3.17), separated by a stationary discontinuity.

3.3. Two intermediate states approximate Riemann solver. We turn in this section to the precise definition of the proposed approximate Riemann solver. We adopt a simple approximate Riemann solver made of three discontinuities propagating with velocities $\lambda_{L}, 0$ and $\lambda_{R}$ and two intermediate states $\mathbf{w}_{L}^{*}$ and $\mathbf{w}_{R}^{*}$, namely:

$$
\mathbf{w}_{\mathcal{R}}\left(\frac{x}{t} ; \mathbf{v}_{L}, \mathbf{v}_{R}\right)=\left\{\begin{aligned}
\mathbf{w}_{L} & \text { if } \frac{x}{t}<\lambda_{L} \\
\mathbf{w}_{L}^{*} & \text { if } \lambda_{L}<\frac{x}{t}<0, \\
\mathbf{w}_{R}^{*} & \text { if } 0<\frac{x}{t}<\lambda_{R}, \\
\mathbf{w}_{R} & \text { if } \frac{x}{t}>\lambda_{R}
\end{aligned}\right.
$$

with $\lambda_{L}<0$ and $\lambda_{R}>0$ (see Figure 3.1). The speeds of propagation $\lambda_{L}$ and $\lambda_{R}$ and the intermediate states $\mathbf{w}_{L}^{*}:=\mathbf{w}_{L}^{*}\left(\Delta t, \Delta x ; \mathbf{v}_{L}, \mathbf{v}_{R}\right)$ and $\mathbf{w}_{R}^{*}:=\mathbf{w}_{R}^{*}\left(\Delta t, \Delta x ; \mathbf{v}_{L}, \mathbf{v}_{R}\right)$ have now to be defined. To address such an issue, we will impose several suitable 


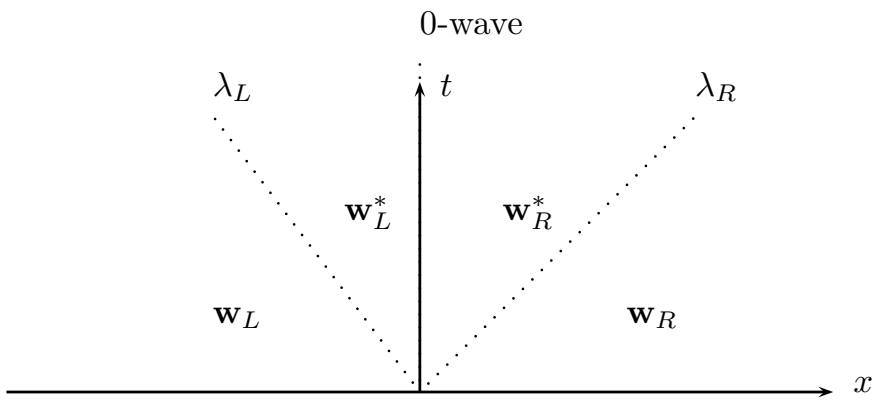

FIG. 3.1. Simple approximate Riemann solver for the shallow water equations.

relations to get the intermediate states as function of the characteristic speeds. Next, we will fix $\lambda_{L, R}$ in order to statisfy some robustness properties.

At this level, we focus on the evaluation of $\mathbf{w}_{L, R}^{*}$ and then we have four unknowns to solve. As a consequence, four relations are expected. According to Theorem 3.2, we first impose the consistency relations (3.18) which gives here the two relations

$$
\begin{aligned}
& h_{R} u_{R}-h_{L} u_{L}=\lambda_{L}\left(h_{L}^{*}-h_{L}\right)+\lambda_{R}\left(h_{R}-h_{R}^{*}\right), \\
& \left(h_{R} u_{R}^{2}+g \frac{h_{R}^{2}}{2}\right)-\left(h_{L} u_{L}^{2}+g \frac{h_{L}^{2}}{2}\right)+g \Delta x\left\{h \partial_{x} z\right\}\left(\Delta t, \Delta x ; \mathbf{v}_{L}, \mathbf{v}_{R}\right)= \\
& \lambda_{L}\left(h_{L}^{*} u_{L}^{*}-h_{L} u_{L}\right)+\lambda_{R}\left(h_{R} u_{R}-h_{R}^{*} u_{R}^{*}\right),
\end{aligned}
$$

where $\left\{h \partial_{x} z\right\}\left(\Delta t, \Delta x ; \mathbf{v}_{L}, \mathbf{v}_{R}\right)$ denotes a consistent approximation of the source term $h \partial_{x} z$ in the sense of (3.8). For the sake of clarity in the notations, we will omit the arguments to write $\left\{h \partial_{x} z\right\}$. It is worth noticing that the definition of $\left\{h \partial_{x} z\right\}$ is crucial to satisfy the required steady state property. In the following statement, we show that the formula governing $\left\{h \partial_{x} z\right\}$ is fully prescribed by the steady state conditions (3.4)-(3.5)-(3.12) given in Theorem 3.2 (iii).

LEMMA 3.3. Let $\mathbf{v}_{L}$ and $\mathbf{v}_{R}$ be given in $\Omega_{z}$ such that (3.4) and (3.5) hold. To enforce the approximate Riemann solver (3.20) to be stationary, namely $\mathbf{v}_{L}^{*}=\mathbf{v}_{L}$ and $\mathbf{v}_{R}^{*}=\mathbf{v}_{R}$, necessarily we have

$$
\Delta x\left\{h \partial_{x} z\right\}=\frac{h_{L} h_{R}}{\bar{h}}\left(z_{R}-z_{L}\right)-\frac{\left(h_{R}-h_{L}\right)^{3}}{4 \bar{h}},
$$

where we have set $\bar{h}=\frac{h_{L}+h_{R}}{2}$. Let us underline from now on that similar topography source term averages can be found in the litterature, see for instance formula (3.78) in $[33]$.

Proof. Let us enforce the approximate Riemann solver to be stationary to write

$$
h_{L}^{*}=h_{L}, \quad h_{L}^{*} u_{L}^{*}=h_{L} u_{L}, \quad h_{R}^{*}=h_{R}, \quad h_{R}^{*} u_{R}^{*}=h_{R} u_{R} .
$$

Since (3.4) is satisfied, (3.21) immediately holds true. Concerning (3.22), we get

$$
\left(h_{R} u_{R}^{2}+g \frac{h_{R}^{2}}{2}\right)-\left(h_{L} u_{L}^{2}+g \frac{h_{L}^{2}}{2}\right)+g \Delta x\left\{h \partial_{x} z\right\}=0,
$$

to obtain $-g \Delta x\left\{h \partial_{x} z\right\}=\frac{\left(h_{R} u_{R}\right)^{2}}{h_{R}}-\frac{\left(h_{L} u_{L}\right)^{2}}{h_{L}}+\frac{g}{2}\left(h_{R}+h_{L}\right)\left(h_{R}-h_{L}\right)$. Arguing (3.4), 
we have the following sequence of equalities:

$$
\begin{aligned}
-g \Delta x\left\{h \partial_{x} z\right\} & =q^{2}\left(\frac{1}{h_{R}}-\frac{1}{h_{L}}\right)+\frac{g}{2}\left(h_{R}+h_{L}\right)\left(h_{R}-h_{L}\right), \\
& =-\frac{q^{2}}{h_{L} h_{R}}\left(h_{R}-h_{L}\right)+\frac{g}{2}\left(h_{R}+h_{L}\right)\left(h_{R}-h_{L}\right), \\
& =\frac{g}{2}\left(h_{R}^{2}-h_{L}^{2}\right)-\frac{q^{2}\left(h_{R}^{2}-h_{L}^{2}\right)}{h_{L} h_{R}\left(h_{L}+h_{R}\right)} .
\end{aligned}
$$

But from (3.5), we write

$$
-g\left(z_{R}-z_{L}\right)=g\left(h_{R}-h_{L}\right)-\frac{q^{2}}{2 h_{L}^{2} h_{R}^{2}}\left(h_{R}^{2}-h_{L}^{2}\right),
$$

so that

$$
-\frac{q^{2}\left(h_{R}^{2}-h_{L}^{2}\right)}{h_{L} h_{R}}=-2 g h_{L} h_{R}\left(z_{R}-z_{L}+h_{R}-h_{L}\right) .
$$

We finally obtain

$$
-g \Delta x\left\{h \partial_{x} z\right\}=\frac{g}{2}\left(h_{R}^{2}-h_{L}^{2}\right)-2 g \frac{h_{L} h_{R}}{h_{L}+h_{R}}\left(z_{R}-z_{L}+h_{R}-h_{L}\right)
$$

that is to say

$$
\Delta x\left\{h \partial_{x} z\right\}=\frac{h_{L} h_{R}}{\bar{h}}\left(z_{R}-z_{L}\right)+\left(h_{R}-h_{L}\right)\left(\frac{h_{L} h_{R}}{\bar{h}}-\bar{h}\right),
$$

and the required defintion of $\left\{h \partial_{x} z\right\}$ is achieved.

It is clear that the definition (3.23) for the source term approximation is consistent in the sense (3.8) since we have $\lim _{\substack{\mathbf{v}_{L}, \mathbf{v}_{R} \rightarrow \mathbf{v} \\ \Delta x \rightarrow 0}}\left\{h \partial_{x} z\right\}=h \partial_{x} z$. However, this defintion is not fully relevant. Indeed, assume $z_{L}=z_{R}$ so that the source term naturally vanishes. As a consequence, we can expect the approximation of the source term to be an approximation of zero as follows:

$$
\left\{h \partial_{x} z\right\}_{\left.\right|_{\left\{z_{L}=z_{R}\right\}}}=\varepsilon(\Delta x) \stackrel{\Delta x \rightarrow 0}{\longrightarrow} 0 .
$$

Of course, such property is not satisfied by (3.23). Therefore, we propose to introduce:

$$
\delta h=\left\{\begin{array}{lr}
h_{R}-h_{L}, & \text { if }\left|h_{R}-h_{L}\right| \leq C \Delta x \\
\operatorname{sign}\left(h_{R}-h_{L}\right) C \Delta x, & \text { otherwise, }
\end{array}\right.
$$

where $C$ denotes some $L^{\infty}$-bound of the $x$-derivative of $h$ in the region where $h$ admits a $x$-derivative. Next, we suggest to consider the following approximation:

$$
\Delta x\left\{h \partial_{x} z\right\}=\frac{h_{L} h_{R}}{\bar{h}}\left(z_{R}-z_{L}\right)+\frac{\delta h^{3}}{4 \bar{h}} .
$$

By involving such modified source term approximation, we immediately note that the necessary condition stated Lemma 3.3 holds true as soon as the steady state under consideration is smooth and satisfies (3.3).

Since $\left\{h \partial_{x} z\right\}$ is prescribed, we have to determine $h_{L, R}^{*}$ and $u_{L, R}^{*}$. From (3.21)(3.22), two relations are known. We suggest to consider the continuity of the Riemann invariants $I_{0}^{(1)}$ and $I_{0}^{(2)}$ across the stationary wave to get the two missing relations. 
4. A positive fully well-balanced approximate Riemann solver. The first idea we propose is based on a suitable linearization of the Riemann invariants $I_{0}^{(1,2)}$ which makes the intermediate states easily solvable. In addition, by enforcing the characteristic speed to be large enough, $\left|\lambda_{L, R}\right|>>1$, we will show that the obtained approximate Riemann solver is positivity preserving, namely $h_{L, R}^{*}>0$.

We write the continuity of $I_{0}^{(1,2)}$ as follows:

$$
\begin{aligned}
& h_{L}^{*} u_{L}^{*}=h_{R}^{*} u_{R}^{*}, \\
& h_{L}^{*} \frac{u_{L}^{2}}{2 h_{L}}+g\left(h_{L}^{*}+z_{L}\right)=h_{R}^{*} \frac{u_{R}^{2}}{2 h_{R}}+g\left(h_{R}^{*}+z_{R}\right) .
\end{aligned}
$$

To simplify the notation, let us set

$$
\begin{aligned}
& h^{H L L}=\frac{\lambda_{R} h_{R}-\lambda_{L} h_{L}}{\lambda_{R}-\lambda_{L}}-\frac{1}{\lambda_{R}-\lambda_{L}}\left(h_{R} u_{R}-h_{L} u_{L}\right), \\
& \left.q^{H L L}=\frac{\lambda_{R} h_{R} u_{R}-\lambda_{L} h_{L} u_{R}}{\lambda_{R}-\lambda_{L}}-\frac{1}{\lambda_{R}-\lambda_{L}}\left(\left(h_{R} u_{R}^{2}+g \frac{h_{R}^{2}}{2}\right)-\left(h_{L} u_{L}^{2}+g \frac{h_{L}^{2}}{2}\right)\right) 4.4\right)
\end{aligned}
$$

which is nothing but the intermediate state involved by the well-known HLL scheme [25] when approximating the homogeneous shallow-water equations.

Now, we solve the system made of (3.21)-(3.22)-(4.1)-(4.2) to obtain:

$$
\begin{aligned}
q^{*} & =h_{L}^{*} u_{L}^{*}=h_{R}^{*} u_{R}^{*}=q^{H L L}-\frac{g}{\lambda_{R}-\lambda_{L}} \Delta x\left\{h \partial_{x} z\right\}, \\
h_{L}^{*} & =\frac{\left(\lambda_{R}-\lambda_{L}\right)\left(g+\frac{u_{R}^{2}}{2 h_{R}}\right) h^{H L L}+g \lambda_{R}\left(z_{R}-z_{L}\right)}{\lambda_{R}\left(g+\frac{u_{L}^{2}}{2 h_{L}}\right)-\lambda_{L}\left(g+\frac{u_{R}^{2}}{2 h_{R}}\right)}, \\
h_{R}^{*} & =\frac{\left(\lambda_{R}-\lambda_{L}\right)\left(g+\frac{u_{L}^{2}}{2 h_{L}}\right) h^{H L L}-g \lambda_{L}\left(z_{L}-z_{R}\right)}{\lambda_{R}\left(g+\frac{u_{L}^{2}}{2 h_{L}}\right)-\lambda_{L}\left(g+\frac{u_{R}^{2}}{2 h_{R}}\right)},
\end{aligned}
$$

these equations being complemented by (3.24)-(3.25). This approximate Riemann solver satisfies the following statement:

Lemma 4.1. There exists $-\lambda_{L}>0$ and $\lambda_{R}>0$ large enough such that $h_{L}^{*}$ and $h_{R}^{*}$ are positive. Moreover, as soon as $\mathbf{v}_{L}$ and $\mathbf{v}_{R}$ define a steady state according to (3.3)-(3.4)-(3.5), then $h_{L, R}^{*}=h_{L, R}$ and $u_{L, R}^{*}=u_{L, R}$.

Proof. Concerning the positiveness of the intermediate water depth, let us first assume $-\lambda_{L}$ and $\lambda_{R}$ large enough to enforce $h^{H L L}$ to be positive.

Next, we first assume $z_{R}-z_{L}>0$ so that $h_{L}^{*}$ is obviously positive. Concerning $h_{R}^{*}$ we have

$$
h_{R}^{*}=\frac{\left(1+\left|\frac{\lambda_{L}}{\lambda_{R}}\right|\right)\left(g+\frac{u_{L}^{2}}{2 h_{L}}\right) h^{H L L}+g\left|\frac{\lambda_{L}}{\lambda_{R}}\right|\left(z_{L}-z_{R}\right)}{\left(g+\frac{u_{L}^{2}}{2 h_{L}}\right)+\left|\frac{\lambda_{L}}{\lambda_{R}}\right|\left(g+\frac{u_{R}^{2}}{2 h_{R}}\right)} .
$$

By considering $\left|\frac{\lambda_{L}}{\lambda_{R}}\right|$ small enough, which is always possible, we obtain $h_{R}^{*}>0$. Similarly, if $z_{R}-z_{L}<0$, we have $h_{R}^{*}>0$ and we get $h_{L}^{*}>0$ as soon as $\left|\frac{\lambda_{R}}{\lambda_{L}}\right|$ is fixed small enough. To establish the stationary property, let us first observe that since (3.3) 
holds, the definition (3.24)-(3.25) of $\left\{h \partial_{x} z\right\}$ boils down to (3.23). Next, we plug (3.4) and (3.5) inside (4.5)-(4.6)-(4.7) to easily obtain

$$
h_{L}^{*}=h_{L}, \quad h_{R}^{*}=h_{R}, \quad u_{L}^{*}=u_{L}, \quad u_{R}^{*}=u_{R} .
$$

Let us show for instance in details that $h_{L}^{*}=h_{L}$. First and since (3.4) hold true, it is clear that $h^{H L L}$ is given by

$$
\left(\lambda_{R}-\lambda_{L}\right) h^{H L L}=\lambda_{R} h_{R}-\lambda_{L} h_{L} .
$$

Using this identity in the numerator of (4.6) that we call $N$ gives

$$
\begin{aligned}
N & =\left(\lambda_{R} h_{R}-\lambda_{L} h_{L}\right)\left(g+\frac{u_{R}^{2}}{2 h_{R}}\right)+g \lambda_{R}\left(z_{R}-z_{L}\right) \\
& =\lambda_{R} h_{R}\left(g+\frac{u_{R}^{2}}{2 h_{R}}\right)-\lambda_{L} h_{L}\left(g+\frac{u_{R}^{2}}{2 h_{R}}\right)+g \lambda_{R}\left(z_{R}-z_{L}\right) .
\end{aligned}
$$

Now using (3.5) leads to

$$
\begin{aligned}
N & =\lambda_{R} h_{R}\left(g+\frac{u_{R}^{2}}{2 h_{R}}\right)-\lambda_{L} h_{L}\left(g+\frac{u_{R}^{2}}{2 h_{R}}\right)+g \lambda_{R}\left(z_{R}-z_{L}\right) \\
& =\lambda_{R}\left(g h_{R}+\frac{u_{R}^{2}}{2}\right)-\lambda_{L} h_{L}\left(g+\frac{u_{R}^{2}}{2 h_{R}}\right)+g \lambda_{R}\left(z_{R}-z_{L}\right) \\
& =\lambda_{R}\left(g h_{L}+\frac{u_{L}^{2}}{2}-g\left(z_{R}-z_{L}\right)\right)-\lambda_{L} h_{L}\left(g+\frac{u_{R}^{2}}{2 h_{R}}\right)+g \lambda_{R}\left(z_{R}-z_{L}\right) \\
& =\lambda_{R} h_{L}\left(g+\frac{u_{L}^{2}}{2 h_{L}}\right)-\lambda_{L} h_{L}\left(g+\frac{u_{R}^{2}}{2 h_{R}}\right),
\end{aligned}
$$

which is nothing but $h_{L}$ times the denominator of $h_{L}^{*}$ so that $h_{L}^{*}=h_{L}$. Similarly, we get $h_{R}^{*}=h_{R}$. Let us now show in details that $q^{*}=h_{L} u_{L}=h_{R} u_{R}$. Let us first recall that $h_{L} u_{L}=h_{R} u_{R}$ by (3.4). We have seen in the proof of Lemma 3.3 that the definition (3.23) is equivalent to

$$
-g \Delta x\left\{h \partial_{x} z\right\}=\frac{\left(h_{R} u_{R}\right)^{2}}{h_{R}}-\frac{\left(h_{L} u_{L}\right)^{2}}{h_{L}}+\frac{g}{2}\left(h_{R}+h_{L}\right)\left(h_{R}-h_{L}\right),
$$

which also writes

$$
\left(h_{R} u_{R}^{2}+g \frac{h_{R}^{2}}{2}\right)-\left(h_{L} u_{L}^{2}+g \frac{h_{L}^{2}}{2}\right)+g \Delta x\left\{h \partial_{x} z\right\}=0,
$$

provided that (3.4) and (3.4) are valid (which is the case here). This last equality says that the left-hand side in (3.22) equals zero, so that we immediately get by $(3.22)$ that $q^{*}=h_{L} u_{L}=h_{R} u_{R}$. The proof is completed.

Since the topography function is smooth, we formally have $\left|z_{R}-z_{L}\right|=\mathcal{O}(\Delta x)$. As a consequence, let us emphasize that, as soon as the mesh is fine enough, the restrictions to be put on $\lambda_{L}$ and $\lambda_{R}$ will be very weak.

Now, by applying Theorem 3.2, the finite volume scheme (3.19) associated with the approximate Riemann solver (3.20)-(4.5)-(4.6)-(4.7) is positivity preserving and fully well-balanced.

5. Nonlinear two intermediate states approximate Riemann solver. In the second approximate Riemann solver we present, we suggest to impose the continuity of the Riemann invariants $I_{0}^{(1,2)}$ across the stationary wave without imposing any linearization. Hence, the two consistency relations (3.21) and (3.22) are now supplemented by

$$
\begin{aligned}
& q^{*}:=h_{L}^{*} u_{L}^{*}=h_{R}^{*} u_{R}^{*}, \\
& \frac{\left(u_{L}^{*}\right)^{2}}{2}+g\left(h_{L}^{*}+z_{L}\right)=\frac{\left(u_{R}^{*}\right)^{2}}{2}+g\left(h_{R}^{*}+z_{R}\right) .
\end{aligned}
$$


Let us first observe that $q^{*}$ is easily obtained by involving (3.22) and (5.1) which gives

$$
q^{*}=q^{H L L}-\frac{g}{\lambda_{R}-\lambda_{L}} \Delta x\left\{h \partial_{x} z\right\},
$$

where $q^{H L L}$ is defined by (4.4).

It then remains to define $h_{L}^{*}$ and $h_{R}^{*}$ by solving the system (3.21) and (5.2):

$$
\left\{\begin{array}{l}
\lambda_{L}\left(h_{L}^{*}-h_{L}\right)+\lambda_{R}\left(h_{R}-h_{R}^{*}\right)-\left(q_{R}-q_{L}\right)=0 \\
\frac{\left(q^{*}\right)^{2}}{2}\left(\frac{1}{\left(h_{L}^{*}\right)^{2}}-\frac{1}{\left(h_{R}^{*}\right)^{2}}\right)+g\left(h_{L}^{*}-h_{R}^{*}\right)-g\left(z_{R}-z_{L}\right)=0
\end{array}\right.
$$

where we have set $q_{L}=h_{L} u_{L}$ and $q_{R}=h_{R} u_{R}$. Defining the functions

$$
\begin{aligned}
& y(x)=\frac{\lambda_{L}}{\lambda_{R}}\left(x-h_{L}\right)+h_{R}-\frac{q_{R}-q_{L}}{\lambda_{R}}, \\
& \varphi\left(x, y ; q^{*}\right)=\frac{\left(q^{*}\right)^{2}}{2 g}\left(\frac{1}{x^{2}}-\frac{1}{y^{2}}\right)+(x-y)-\left(z_{R}-z_{L}\right),
\end{aligned}
$$

it clearly amounts to define $h_{L}^{*}$ as the solution of

$$
\varphi\left(x, y(x) ; q^{*}\right)=0
$$

$h_{R}^{*}$ being then simply given by $h_{R}^{*}=y\left(h_{L}^{*}\right)$. Because it will be useful in the sequel, let us remark the following identity satisfied by the linear function $y$ :

$$
y\left(h^{H L L}\right)=h^{H L L},
$$

where $h^{H L L}$ is defined by (4.3).

Our main goal is now to study the function $x \mapsto \varphi\left(x, y(x) ; q^{*}\right)$ and its roots.

5.1. The case $q^{*}=0$. Let us notice that the case $q^{*}=0$, which will be useful in the following, can be explicitly solved. Denoting by $h_{L}^{0 *}$ and $h_{R}^{0 *}=y\left(h_{L}^{0 *}\right)$ the values of $h_{L}^{*}$ and $h_{R}^{*}$ in this particular case, we easily find

$$
\left\{\begin{array}{l}
h_{L}^{0 *}=h^{H L L}+\alpha\left(z_{R}-z_{L}\right) \\
h_{R}^{0 *}=h^{H L L}-(1-\alpha)\left(z_{R}-z_{L}\right)
\end{array}\right.
$$

where we have set $\alpha=\frac{\lambda_{R}}{\lambda_{R}-\lambda_{L}} \in(0,1)$.

About the definition of $\lambda_{L}$ and $\lambda_{R}$. We can notice from now on that provided that $\lambda_{R}>0$ and $-\lambda_{L}>0$ are chosen sufficiently large, $h^{H L L}, h_{L}^{0 *}$ and $h_{R}^{0 *}$ can be given positive values. Indeed, the quantity

$$
\frac{q_{R}-q_{L}}{\lambda_{R}-\lambda_{L}}
$$

naturally tends to zero so that $h^{H L L}$ behaves like a convex combination of $h_{L}>0$ and $h_{R}>0$. On the other hand, we have $h_{L}^{0 *}>0$ and $h_{R}^{0 *}>0$ as soon as

$$
\left\{\begin{array}{l}
\alpha h_{R}+(1-\alpha) h_{L}>-\alpha\left(z_{R}-z_{L}\right) \\
\alpha h_{R}+(1-\alpha) h_{L}>(1-\alpha)\left(z_{R}-z_{L}\right) .
\end{array}\right.
$$


This naturally holds true in the following asymptotic regimes associated with sufficiently large values of $-\lambda_{L}$ and $\lambda_{R}$, namely

$$
\left\{\begin{array}{l}
\alpha \sim 1 \quad \text { if } \quad\left(z_{R}-z_{L}\right)>0 \\
\alpha \sim 0 \quad \text { if } \quad\left(z_{R}-z_{L}\right)<0
\end{array}\right.
$$

Importantly, these asymptotic definitions of $\lambda_{L}$ and $\lambda_{R}$ will play an crucial role in the forthcoming developements and will be adopted in the rest of the paper.

Remark. The natural choice $\lambda_{R}=-\lambda_{L}$ (related to the Rusanov scheme [37]) gives $\alpha=1 / 2$ which does not correspond to any of these asymptotic regimes.

Remark. By the definition of $h^{H L L}$, we have

$$
h^{H L L}=\frac{-\lambda_{L}+u_{L}}{\lambda_{R}-\lambda_{L}} h_{L}+\frac{\lambda_{R}-u_{R}}{\lambda_{R}-\lambda_{L}} h_{R}
$$

so that $h^{H L L}$ is clearly positive provided that $\lambda_{L}<u_{L}$ and $\lambda_{R}>u_{R}$, which are natural conditions on $\lambda_{L}$ and $\lambda_{R}$ (in particular, these conditions are satisifed provided that $\lambda_{R}>0$ and $-\lambda_{L}>0$ are chosen sufficiently large). In other words, there is no need of asymptotic condition on $\frac{q_{R}-q_{L}}{\lambda_{R}-\lambda_{L}}$ to prove that $h^{H L L}$ is positive.

5.2. The case $q^{*} \neq 0$. Our objective is now to prove that under the above asymptotic definitions (5.7) to be satisfied by both $\lambda_{L}$ and $\lambda_{R}$, there exists at least one positive root $x$ to

$$
\psi(x):=\varphi\left(x, y(x) ; q^{*}\right)=0
$$

and such that $y(x)>0$. This will ensure that $h_{L}^{*}$ and $h_{R}^{*}$ are positive.

First, let us observe that $y(x)>0$ yields to the constraint $x \in\left(0, x_{0}\right)$ with $x_{0}$ defined by

$$
x_{0}=\frac{\lambda_{R} h_{R}-\lambda_{L} h_{L}}{-\lambda_{L}}-\frac{q_{R}-q_{L}}{-\lambda_{L}}=\frac{1}{1-\alpha} h^{H L L} .
$$

Since $\lambda_{L, R}$ are large enough to ensure $h^{H L L}$ positive, then we have $x_{0}>0$. Moreover, with $h^{H L L}=(1-\alpha) x_{0}$ we get the following estimation : $0<h^{H L L}<x_{0}$. The line $x \in \mathbb{R}$ can thus be decomposed into three parts, namely $x<0$ for which $y(x)>0$, $0<x<x_{0}$ which ensures $y(x)>0$, and at last $x>x_{0}$ giving $y(x)<0$. We will be naturally more focused on the region $0<x<x_{0}$ with $y(x)>0$. Note in passing that $y(0)=y_{0}:=\frac{1}{\alpha} h^{H L L}>0$ while $y\left(x_{0}\right)=0$.

More precisely, our objective is to prove that the graph of the function $\psi$ is given by the Figure 5.1 below, so that in particular $\psi(x)=0$ admits three roots of possible interest in between 0 and $x_{0}$.

Let us first state some basic properties, the proofs of which being left to the reader.

Lemma 5.1. The functions $y: \mathbb{R} \rightarrow \mathbb{R}$ defined by (5.4), $\varphi: \mathbb{R} \times \mathbb{R} \rightarrow \mathbb{R}$ defined 


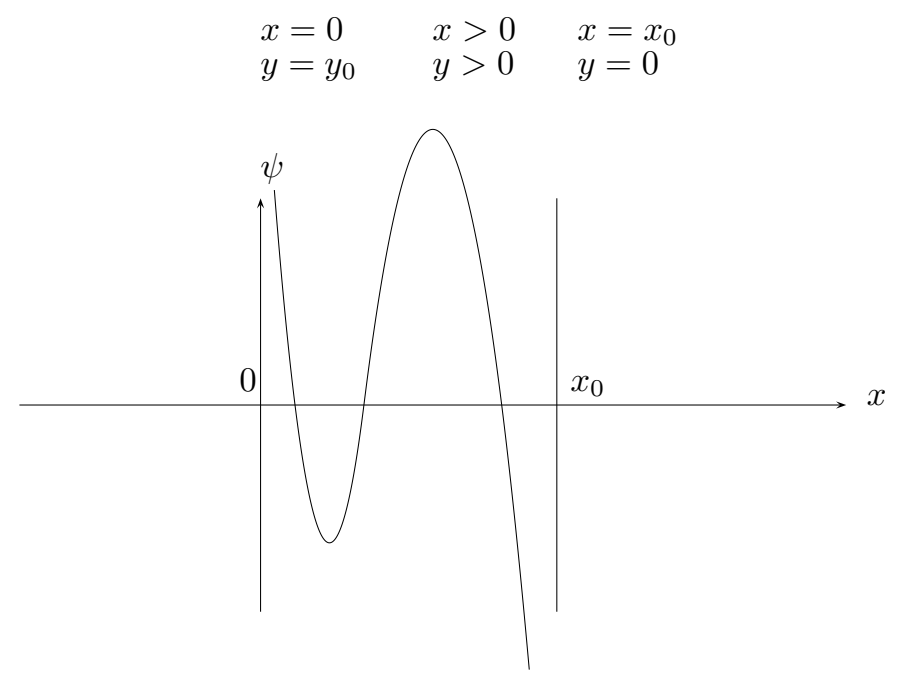

FIG. 5.1. Function $\psi$ for sufficiently large values of $-\lambda_{L}$ and $\lambda_{R}$ under the asymptotic definitions (5.7)

by (5.5) and $\psi: \mathbb{R} \rightarrow \mathbb{R}$ defined by $\psi(x)=\varphi\left(x, y(x) ; q^{*}\right)$ satisfy

$$
\left\{\begin{array}{l}
y^{\prime}(x)=\frac{\lambda_{L}}{\lambda_{R}}<0 \\
\psi^{\prime}(x)=\frac{\left(q^{*}\right)^{2}}{g}\left(\frac{y^{\prime}(x)}{y(x)^{3}}-\frac{1}{x^{3}}\right)+\left(1-y^{\prime}(x)\right), \\
\psi^{\prime \prime}(x)=\frac{3\left(q^{*}\right)^{2}}{g}\left(\frac{1}{x^{4}}-\frac{y^{\prime}(x)^{2}}{y(x)^{4}}\right)
\end{array}\right.
$$

and

$$
\begin{aligned}
& \lim _{x \rightarrow 0^{-}} \psi(x)=+\infty, \quad \lim _{x \rightarrow x_{0}^{-}} \psi(x)=-\infty, \quad \lim _{x \rightarrow-\infty} \psi(x)=-\infty, \\
& \lim _{x \rightarrow 0^{+}} \psi(x)=+\infty, \quad \lim _{x \rightarrow x_{0}^{+}} \psi(x)=-\infty, \quad \lim _{x \rightarrow+\infty} \psi(x)=+\infty,
\end{aligned}
$$

Moreover, at most the function $\psi$ admits five roots.

From the properties stated in this lemma, it is first clear that

$$
\lim _{x \rightarrow 0^{+}} \psi^{\prime \prime}(x)=+\infty \text { and } \lim _{x \rightarrow 0^{-}} \psi^{\prime \prime}(x)=-\infty,
$$

then we get $\psi^{\prime \prime}(x)>0$ for all $x>0$ in a neighborhood of zero while $\psi^{\prime \prime}(x)<0$ for all $x<x_{0}$ in a neighborhood of $x_{0}$. Similarly, we have

$$
\lim _{x \rightarrow 0^{+}} \psi^{\prime}(x)=-\infty \text { and } \lim _{x \rightarrow 0^{-}} \psi^{\prime \prime}(x)=-\infty,
$$

to obtain $\psi^{\prime}(x)<0$ in the neighborhoods of both 0 and $x_{0}$. 
In order to prove that the function $\psi$ admits three roots of possible interest in between 0 and $x_{0}$, it is then a matter to prove that there exists two values $x_{-}$and $x_{+}$in the interval $\left(0, x_{0}\right)$, such that $x_{-}<x_{+}$and

$$
\psi\left(x_{-}\right)<0 \text { and } \psi\left(x_{+}\right)<0 .
$$

The definition of these values will depend on the sign of $\Delta z:=z_{R}-z_{L}$.

Towards the definitions of $x_{-}$and $x_{+}$. Let us first calculate $\psi\left(h_{L}^{0 *}\right)$ and $\psi\left(h_{R}^{0 *}\right)$. Since by definition we clearly have $h_{L}^{0 *}-h_{R}^{0 *}=\Delta z$, it comes

$$
\psi\left(h_{L}^{0 *}\right)=\frac{\left(q^{*}\right)^{2}}{2 g}\left(\frac{1}{\left(h_{L}^{0 *}\right)^{2}}-\frac{1}{\left(h_{R}^{0 *}\right)^{2}}\right)=-\frac{1}{2 g}\left(\frac{q^{*}}{h_{L}^{0 *} h_{R}^{0 *}}\right)^{2}\left(h_{L}^{0 *}+h_{R}^{0 *}\right) \Delta z,
$$

which indicates that the $\operatorname{sign}$ of $\psi\left(h_{L}^{0 *}\right)$ is given by the sign of $-\Delta z$, at least for sufficiently large values of $-\lambda_{L}$ and $\lambda_{R}$ in the regime (5.7) to enforce $h_{L, R}^{0 *}>0$.

The case $\Delta z>0$. On the first hand, we have $\psi\left(h_{L}^{0 *}\right)<0$ so that we propose to set

$$
x_{-}=h_{L}^{0 *}=h^{H L L}+\alpha \Delta z .
$$

The point $x_{+}$is defined as a small perturbation of $x_{-}$given by

$$
x_{+}=x_{-}+\alpha\left(q^{*}\right)^{2} \varepsilon \Delta z=h^{H L L}+\alpha \Delta z\left(1+\left(q^{*}\right)^{2} \varepsilon\right),
$$

for a sufficiently small value of $\varepsilon>0$ such that $x_{-}<x_{+}<x_{0}$. Now, we have to fix the parameter $\varepsilon$. By definition of $x_{+}$and since $y\left(h^{H L L}\right)=h^{H L L}$, we immediately deduce that that

$$
y\left(x_{+}\right)=h^{H L L}+\frac{\lambda_{L}}{\lambda_{R}} \alpha \Delta z\left(1+\left(q^{*}\right)^{2} \varepsilon\right)
$$

that is to say

$$
y\left(x_{+}\right)=h^{H L L}+(\alpha-1) \Delta z\left(1+\left(q^{*}\right)^{2} \varepsilon\right) .
$$

In particular, we note that

$$
\begin{aligned}
& y\left(x_{+}\right)-x_{+}=-\Delta z\left(1+\left(q^{*}\right)^{2} \varepsilon\right), \\
& y\left(x_{+}\right)+x_{+}=2 h^{H L L}+(2 \alpha-1) \Delta z\left(1+\left(q^{*}\right)^{2} \varepsilon\right) .
\end{aligned}
$$

By definition of $\psi$, we thus clearly have

$$
\psi\left(x_{+}\right)=-\frac{\left(q^{*}\right)^{2}}{2 g x_{+}^{2}\left(y\left(x_{+}\right)\right)^{2}}\left(2 h^{H L L}+(2 \alpha-1) \Delta z\left(1+\left(q^{*}\right)^{2} \varepsilon\right)\right) \Delta z\left(1+\left(q^{*}\right)^{2} \varepsilon\right)+\Delta z\left(q^{*}\right)^{2} \varepsilon .
$$

But recall that in the case $\Delta z>0,-\lambda_{L}$ and $\lambda_{R}$ are defined large enough according to (5.7) so that $\alpha \sim 1$, or equivalently $\frac{-\lambda_{L}}{\lambda_{R}} \sim 0$, which gives in particular

$q^{*} \sim q_{R}, \quad h^{H L L} \sim h_{R}, \quad x_{-} \sim h_{R}+\Delta z, \quad x_{+} \sim h_{R}+\Delta z\left(1+\left(q_{R}\right)^{2} \varepsilon\right), \quad y\left(x_{+}\right) \sim h_{R}$.

Then,

$$
\begin{aligned}
\psi\left(x_{+}\right) \sim & -\frac{q_{R}^{2} \Delta z}{2 g h_{R}^{2}\left(h_{R}+\Delta z\left(1+\left(q_{R}\right)^{2} \varepsilon\right)\right)^{2}} \times \\
& \left(\left(2 h_{R}+\Delta z\left(1+\left(q_{R}\right)^{2} \varepsilon\right)\right)\left(1+\left(q_{R}\right)^{2} \varepsilon\right)-2 g h_{R}^{2}\left(h_{R}+\Delta z\left(1+\left(q_{R}\right)^{2} \varepsilon\right)\right)^{2} \varepsilon\right) .
\end{aligned}
$$


Now setting $\varepsilon=\frac{1}{2 g h_{R}^{2} \Delta z}$ allows to cancel all the terms involving the quantity ( $1+$ $\left.\left(q_{R}\right)^{2} \varepsilon\right)$ in the brackets above so as to obtain

$$
\left(2 h_{R}+\Delta z\left(1+\left(q_{R}\right)^{2} \varepsilon\right)\right)\left(1+\left(q_{R}\right)^{2} \varepsilon\right)-2 g h_{R}^{2}\left(h_{R}+\Delta z\left(1+\left(q_{R}\right)^{2} \varepsilon\right)\right)^{2} \varepsilon \sim-\frac{h_{R}^{2}}{\Delta z}
$$

which clearly ensures $\psi\left(x_{+}\right)$to stay in a neighborhood of a positive value. It just remains to prove that with such a definition of $\varepsilon, x_{+}<x_{0}$ that is to say to prove the following inequality:

$$
h^{H L L}+\alpha \Delta z\left(1+\left(q^{*}\right)^{2} \varepsilon\right)<\frac{1}{1-\alpha} h^{H L L} .
$$

This estimation is clearly true in the asymptotic regime $\alpha \sim 1$ since the right hand side goes to $+\infty$ and the left hand side is bounded.

The case $\Delta z<0$. On the first hand, we have $\psi\left(h_{L}^{0 *}\right)>0$ so that we propose to set

$$
x_{+}=h_{L}^{0 *}=h^{H L L}+\alpha \Delta z .
$$

As before, the point $x_{-}$will be defined as a small perturbation of $x_{+}$, parametrized by $\varepsilon>0$ and defined by

$$
\begin{aligned}
x_{-} & =x_{+}+\alpha\left(q^{*}\right)^{2} \varepsilon \Delta z, \\
& =h^{H L L}+\alpha \Delta z\left(1+\left(q^{*}\right)^{2} \varepsilon\right),
\end{aligned}
$$

for a sufficiently small value of $\varepsilon$ such that $x_{-}<x_{+}<x_{0}$. Very similar calculations as in the case $\Delta z>0$ show that the choice $\varepsilon=-\frac{1}{2 g h_{L}^{2} \Delta z}>0$ is suitable.

In both cases $\Delta z>0$ and $\Delta z<0$, we have thus proved that the function $\psi$ has the shape given by Figure 5.1 for sufficiently large values of $-\lambda_{L}$ and $\lambda_{R}$ defined according to (5.7). In the following, we will denote $h_{1}^{*}, h_{2}^{*}$ and $h_{3}^{*}$ the three roots of (5.8) in $\left(0, x_{0}\right)$ and such that $h_{1}^{*}<h_{2}^{*}<h_{3}^{*}$.

5.3. Characterization of the approximate Riemann solver. We now propose the full characterization of the two intermediate states. The evaluation of $h_{L}^{*} u_{L}^{*}$ and $h_{R}^{*} u_{R}^{*}$ are given by (5.3). Concerning the values of $h_{L}^{*}$ and $h_{R}^{*}$, as soon as $q^{*}=0$, we have $h_{L}^{*}=h_{L}^{0 *}$ and $h_{R}^{*}=h_{R}^{0 *}$ where $h_{L, R}^{0 *}$ are defined by (5.6).

The main difficulty in the evaluation of $h_{L, R}^{*}$ comes from the case $q^{*} \neq 0$. One has to select $h_{L}^{*}$ between the three admissible roots of $(5.8), 0<h_{1}^{*}<h_{2}^{*}<h_{3}^{*}<x_{0}$, previously detailed. The choice is dictated by the preservation of the required fully well-balanced property.

Let us consider $\mathbf{v}_{L}$ and $\mathbf{v}_{R}$ to satisfy the steady state conditions (3.3)-(3.4)-(3.5). Since (3.3) is verified, the source term approximation $\left\{h \partial_{x} z\right\}$ is given by (3.23). Next, arguing (3.4) and (3.5), we easily obtain

$$
q^{*}=h_{L} u_{L}=h_{R} u_{R}
$$

Now, we notice that the steady state conditions (3.4)-(3.5) make $h_{L}$ as a trivial adimissible root of $\psi$. Moreover, we have $y\left(h_{L}\right)=h_{R}$. Hence, we will choose the root of $\psi$ which coincides with $h_{L}$ as soon as (3.4)-(3.5) hold true. To address such an issue, we evaluate the behavior of $\psi$ estimated at $h_{L}$.

Lemma 5.2. Let $\mathbf{v}_{L}$ and $\mathbf{v}_{R}$ in $\Omega$ be given such that (3.3)-(3.4)-(3.5) hold true. We set $0<h_{1}^{*}<h_{2}^{*}<h_{3}^{*}<x_{0}$ the three admissible roots of $\psi$. The root $h_{L}$ satisfies: 
(i) if $\alpha \frac{u_{L}^{2}}{c_{L}^{2}}+(1-\alpha) \frac{u_{R}^{2}}{c_{R}^{2}} \leq 1$ then $h_{L}=h_{2}^{*}$.

(ii) if $\alpha \frac{u_{L}^{2}}{c_{L}^{2}}+(1-\alpha) \frac{u_{R}^{2}}{c_{R}^{2}}>1$ and $\alpha^{2} \frac{u_{L}^{2}}{c_{L}^{4}}-(1-\alpha)^{2} \frac{u_{R}^{2}}{c_{R}^{4}}>0$ then $h_{L}=h_{1}^{*}$.

(iii) if $\alpha \frac{u_{L}^{2}}{c_{L}^{2}}+(1-\alpha) \frac{u_{R}^{2}}{c_{R}^{2}}>1$ and $\alpha^{2} \frac{u_{L}^{2}}{c_{L}^{4}}-(1-\alpha)^{2} \frac{u_{R}^{2}}{c_{R}^{4}}<0$ then $h_{L}=h_{3}^{*}$.

Proof. First, let us assume that both left and right states are sonic point, namely $u_{L}^{2}=c_{L}^{2}$ and $u_{R}^{2}=c_{R}^{2}$, so that (i) holds true with an equality. Since $\mathbf{v}_{L}$ and $\mathbf{v}_{R}$ satisfy (3.4) and (3.5), we easily establish that $\mathbf{v}_{L}=\mathbf{v}_{R}$. Then we enter a very specific defintion of $\psi$ where $h_{2}^{*}=h_{L}=h_{R}$ turns out to be a double root.

Next, according to the analysis of the function $\psi$, we know that the three admissible roots are characterized as follows:

- $h_{1}^{*}$ satisfies $\psi^{\prime}\left(h_{1}^{*}\right)<0$ and $\psi^{\prime \prime}\left(h_{1}^{*}\right)>0$,

- $h_{2}^{*}$ satisfies $\psi^{\prime}\left(h_{2}^{*}\right)>0$,

- $h_{3}^{*}$ satisfies $\psi^{\prime}\left(h_{3}^{*}\right)<0$ and $\psi^{\prime \prime}\left(h_{3}^{*}\right)<0$.

Now, by Lemma 5.1 , we have

$$
\begin{aligned}
& \psi^{\prime}\left(h_{L}\right)=\frac{1}{\alpha}\left(1-\left(\alpha \frac{u_{L}^{2}}{c_{L}^{2}}+(1-\alpha) \frac{u_{R}^{2}}{c_{R}^{2}}\right)\right), \\
& \psi^{\prime \prime}\left(h_{L}\right)=\frac{3 g}{\alpha^{2}}\left(\alpha^{2} \frac{u_{L}^{2}}{c_{L}^{4}}-(1-\alpha)^{2} \frac{u_{R}^{2}}{c_{R}^{4}}\right),
\end{aligned}
$$

and the expected result is obtained.

From now on, let us underline that the following root choice:

(i) if $\alpha \frac{u_{L}^{2}}{c_{L}^{2}}+(1-\alpha) \frac{u_{R}^{2}}{c_{R}^{2}} \leq 1$ then $h_{L}^{*}=h_{2}^{*}$,

(ii) if $\alpha \frac{u_{L}^{2}}{c_{L}^{2}}+(1-\alpha) \frac{u_{R}^{2}}{c_{R}^{2}}>1$ and $\alpha^{2} \frac{u_{L}^{2}}{c_{L}^{4}}-(1-\alpha)^{2} \frac{u_{R}^{2}}{c_{R}^{4}}>0$ then $h_{L}^{*}=h_{1}^{*}$,

(iii) if $\alpha \frac{u_{L}^{2}}{c_{L}^{2}}+(1-\alpha) \frac{u_{R}^{2}}{c_{R}^{2}}>1$ and $\alpha^{2} \frac{u_{L}^{2}}{c_{L}^{4}}-(1-\alpha)^{2} \frac{u_{R}^{2}}{c_{R}^{4}}<0$ then $h_{L}^{*}=h_{3}^{*}$,

would give a complete characterization of the approximate Riemann solver. In addition, the required positiveness and fully well-balanced properties stated in Theorem 3.2 (ii) and (iii) are obviously satisfied.

In fact, to establish the entropy preserving property stated Thorem 3.2 (iii), we need an additional property satisfied by the selected root. Indeed, we can specify the behavior of one of the root in a neighborhood of $z_{L}=z_{R}$, and prove it approaches $h^{H L L}$ as soon as $\left|z_{R}-z_{L}\right|$ is small enough.

Lemma 5.3. Assume $z_{L}=z_{R}$. The three admissible roots satisfy:

(i) If $g\left(h^{H L L}\right)^{3}>\left(q^{*}\right)^{2}$ then $h_{2}^{*}=h^{H L L}$.

(ii) If $g\left(h^{H L L}\right)^{3}<\left(q^{*}\right)^{2}$ and $\alpha>1 / 2$ then $h_{1}^{*}=h^{H L L}$.

(iii) If $g\left(h^{H L L}\right)^{3}<\left(q^{*}\right)^{2}$ and $\alpha<1 / 2$ then $h_{3}^{*}=h^{H L L}$.

Proof. Since $y\left(h^{H L L}\right)=h^{H L L}$, we notice that $h^{H L L}$ is an obvious root of $\psi_{\left.\right|_{\left\{z_{L}=z_{R}\right\}}}$. By evaluating $\psi_{\left.\right|_{\left\{z_{L}=z_{R}\right\}} ^{\prime}}^{\prime}\left(h^{H L L}\right)$ and $\psi_{\left.\right|_{\left\{z_{L}=z_{R}\right\}} ^{\prime \prime}}\left(h^{H L L}\right)$ we get

$$
\psi_{\left.\right|_{\left\{z_{L}=z_{R}\right\}} ^{\prime}}^{\prime}\left(h^{H L L}\right)=\frac{1}{\alpha}\left(1-\frac{\left(q^{*}\right)^{2}}{g\left(h^{H L L}\right)^{3}}\right) \text { and } \psi_{\left.\right|_{\left\{z_{L}=z_{R}\right\}} ^{\prime \prime}}^{\prime \prime}\left(h^{H L L}\right)=\frac{3\left(q^{*}\right)^{2}}{g\left(h^{H L L}\right)^{4}} \frac{2 \alpha-1}{\alpha^{2}},
$$

and the proof is completed by applying the characterization of the admissible roots of $\psi$. $\mathrm{c}$

As a consequence, one of the root of (5.8) is a small perturbation of the root $h^{H L L}$ of $\psi_{\left\{_{\left\{z_{L}=z_{R}\right\}}\right.}$. A precise expansion of this root will be given in the next section. 
We are now able to give the full characterization of the approximate Riemann solver in order for the adopted root to simultaneously satisfy Lemma 5.2 and Lemma 5.3 while the parameter is defined by (5.7). We suggest the following algorithm:

(i) If $z_{R}-z_{L}>0$ and $u_{R}^{2}>c_{R}^{2}$ then $h_{L}^{*}=h_{1}^{*}$,

(ii) If $z_{R}-z_{L}>0$ and $u_{R}^{2}<c_{R}^{2}$ then $h_{L}^{*}=h_{2}^{*}$,

(iii) If $z_{R}-z_{L}<0$ and $u_{L}^{2}<c_{L}^{2}$ then $h_{L}^{*}=h_{2}^{*}$,

(iii) If $z_{R}-z_{L}<0$ and $u_{L}^{2}>c_{L}^{2}$ then $h_{L}^{*}=h_{3}^{*}$.

From (5.7) and arguing Lemma 5.3, it is easy to check that the selected $h_{L}^{*}$ is a perturbation to $h^{H L L}$. Next, by considering $\mathbf{v}_{L}$ and $\mathbf{v}_{R}$ steady states such that (3.3)(3.4)-(3.5) holds, the Lemma 5.2 is verified. As a consequence, we have stated that $h_{L}^{*}$ satisfies the positiveness and the fully well-balanced properties given in Theorem 3.2 (ii) and (iii).

5.4. Discrete entropy inequality. The objective of this section is to prove the validity of a discrete entropy inequality. According to Theorem 3.2 , it is sufficient to prove (3.13) which writes here as follows according to the specific form of the approximate Riemann solver under consideration :

$$
\begin{aligned}
\mathcal{F}\left(\mathbf{w}_{R}\right)-\mathcal{F}\left(\mathbf{w}_{L}\right)+ & \Delta x \sigma_{\nu}\left(\Delta t, \Delta x ; \mathbf{v}_{L}, \mathbf{v}_{R}\right) \leq \\
& \lambda_{L}\left(\mathcal{U}\left(\mathbf{w}_{L}^{*}\right)-\mathcal{U}\left(\mathbf{w}_{L}\right)\right)+\lambda_{R}\left(\mathcal{U}\left(\mathbf{w}_{R}\right)-\mathcal{U}\left(\mathbf{w}_{R}^{*}\right)\right)
\end{aligned}
$$

with $\lim _{\substack{\mathbf{v}_{L}, \mathbf{v}_{R} \rightarrow \mathbf{v} \\ \Delta t, \Delta x \rightarrow 0}} \sigma_{\nu}\left(\Delta t, \Delta x ; \mathbf{v}_{L}, \mathbf{v}_{R}\right)=-g h u \partial_{x} z$. In fact, we will prove a variant of this entropy inequality. More precisely, we will prove this entropy inequality up to a correction term of the form $\Delta x \epsilon(\Delta x)$ with $\epsilon(\Delta x)$ such that $\lim _{\Delta x \rightarrow 0} \epsilon(\Delta x)=0$. This is actually sufficient to get the convergence to an entropy solution according to (a slight variant of) the well-known Lax-Wendroff theorem [20].

THEOREM 5.4. The proposed approximate Riemann solver satisfies the following entropy inequality

$$
\begin{aligned}
\mathcal{F}\left(\mathbf{w}_{R}\right)-\mathcal{F}\left(\mathbf{w}_{L}\right)+ & \Delta x \sigma_{\nu}\left(\Delta t, \Delta x ; \mathbf{v}_{L}, \mathbf{v}_{R}\right)+\Delta x \epsilon(\Delta x) \leq \\
& \lambda_{L}\left(\mathcal{U}\left(\mathbf{w}_{L}^{*}\right)-\mathcal{U}\left(\mathbf{w}_{L}\right)\right)+\lambda_{R}\left(\mathcal{U}\left(\mathbf{w}_{R}\right)-\mathcal{U}\left(\mathbf{w}_{R}^{*}\right)\right)
\end{aligned}
$$

with $\lim _{\substack{\mathbf{v}_{L}, \mathbf{v}_{R} \rightarrow \mathbf{v} \\ \Delta t, \Delta x \rightarrow 0}} \sigma_{\nu}\left(\Delta t, \Delta x ; \mathbf{v}_{L}, \mathbf{v}_{R}\right)=-g h u \partial_{x} z$ and $\lim _{\Delta x \rightarrow 0} \epsilon(\Delta x)=0$.

Proof. Note from now on that in the following and with little abuse in the notations, we will use the same notation $x \rightarrow \epsilon(x)$ for possibly different functions having the property $\lim _{\Delta x \rightarrow 0} \epsilon(\Delta x)=0$ (it will not necessarily represent the same function from a line to another).

The first step of the proof consists in proving that there exists $h_{1}^{*}$ independent of $\Delta z$ such that

$$
h_{L}^{*}=h^{H L L}+h_{1}^{*} \Delta z+\Delta z \epsilon(\Delta z)
$$

and

$$
h_{R}^{*}=h^{H L L}-\frac{1-\alpha}{\alpha} h_{1}^{*} \Delta z+\Delta z \epsilon(\Delta z)
$$

with $\epsilon(\Delta z)$ such that $\lim _{\Delta z \rightarrow 0} \epsilon(\Delta z)=0$. Note that such asymptotic expansions of $h_{L}^{*}$ and $h_{R}^{*}$ are not surprising since we recall that when $z_{L}=z_{R}$, that is to say $\Delta z=0$, 
we have $h_{L}^{*}=h_{R}^{*}=h^{H L L}$. In order to give a rigorous proof, let us first observe that $\varphi\left(x, y(x) ; q^{*}\right)$ defined by (5.5)-(5.4) with $q^{*}$ given by

$$
q^{*}=q^{H L L}-\frac{g}{\lambda_{R}-\lambda_{L}} \Delta x\left\{h \partial_{x} z\right\}
$$

and $\Delta x\left\{h \partial_{x} z\right\}=\frac{h_{L} h_{R}}{\bar{h}}\left(z_{R}-z_{L}\right)+\frac{\delta h^{3}}{4 \bar{h}}$, can be equivalently recast as

$$
\varphi\left(x, y(x) ; q^{*}\right)=\frac{1}{x^{2} y(x)^{2}}\left[p_{0}(x)+p_{1}(x) \Delta z+p_{2}(x)(\Delta z)^{2}\right]
$$

with $q_{0}^{*}=q^{H L L}-\frac{g}{\lambda_{R}-\lambda_{L}} \frac{\delta h^{3}}{4 \bar{h}}$ and

$$
\left\{\begin{array}{l}
p_{0}(x)=\frac{\left(q_{0}^{*}\right)^{2}}{2 g}\left(y(x)^{2}-x^{2}\right)+x^{2} y(x)^{2}(x-y(x)), \\
p_{1}(x)=-\frac{q_{0}^{*}}{\lambda_{R}-\lambda_{L}} \frac{h_{L} h_{R}}{\bar{h}}\left(y(x)^{2}-x^{2}\right)-x^{2} y(x)^{2}, \\
p_{2}(x)=\frac{g}{2}\left(\frac{1}{\lambda_{R}-\lambda_{L}}\right)^{2}\left(\frac{h_{L} h_{R}}{\bar{h}}\right)^{2}\left(y(x)^{2}-x^{2}\right) .
\end{array}\right.
$$

Note that $\varphi\left(x, y(x) ; q^{*}\right)$ and $p_{0}(x)+p_{1}(x) \Delta z+p_{2}(x)(\Delta z)^{2}$ share the same roots. Moreover, as soon as $\Delta z=0$, we have $\varphi\left(h^{H L L}, y\left(h^{H L L}\right) ; q^{*}\right)=p_{0}\left(h^{H L L}\right)=0$ since $y\left(h^{H L L}\right)=h^{H L L}$. From Lemma A.1 in reference [35], the behaviour of the simple root $h_{L}^{*}$ of $\varphi\left(x, y(x) ; q^{*}\right)$ is then given by $(5.11)$ with $h_{1}^{*}$ given by

$$
h_{1}^{*}=-\frac{p_{1}\left(h^{H L L}\right)}{p_{0}^{\prime}\left(h^{H L L}\right)}
$$

which gives after easy calculations

$$
h_{1}^{*}=-\alpha g \frac{\left(h^{H L L}\right)^{3}}{\left(q_{0}^{*}\right)^{2}-g\left(h^{H L L}\right)^{3}} .
$$

Observe that it is always possible to choose $\alpha$ such that the denominator does not have the value zero, at least provided that $\mathbf{v}_{L} \neq \mathbf{v}_{R}$ (the case $\mathbf{v}_{L}=\mathbf{v}_{R}$ does not rise any difficulty and (5.10) is trivially satisfied). Once (5.11) is established, (5.12) follows immediately by definition (5.4) and since $h_{R}^{*}=y\left(h_{L}^{*}\right)\left(\right.$ recall that $h^{H L L}=y\left(h^{H L L}\right)$ ). Before going ahead, let us also notice that regarding $q^{*}=q_{L}^{*}=q_{R}^{*}$ we have

$$
q^{*}=q_{0}^{*}+q_{1}^{*} \Delta z
$$

with

$$
q_{1}^{*}=-\frac{g}{\lambda_{R}-\lambda_{L}} \frac{h_{L} h_{R}}{\bar{h}} \quad \text { and } \quad q_{0}^{*}=q^{H L L}-\frac{g}{\lambda_{R}-\lambda_{L}} \frac{\delta h^{3}}{4 \bar{h}}=q^{H L L}+O\left(\Delta x^{3}\right)
$$

by definition of $\delta h$.

The second step of the proof consists in establishing the asymptotic behaviour of the right-hand side $\Delta \mathcal{U}$ of (5.10) :

$$
\Delta \mathcal{U}=\lambda_{L}\left(\mathcal{U}\left(\mathbf{w}_{L}^{*}\right)-\mathcal{U}\left(\mathbf{w}_{L}\right)\right)+\lambda_{R}\left(\mathcal{U}\left(\mathbf{w}_{R}\right)-\mathcal{U}\left(\mathbf{w}_{R}^{*}\right)\right) .
$$


Let us first calculate $\lambda_{R} \mathcal{U}\left(\mathbf{w}_{R}^{*}\right)-\lambda_{L} \mathcal{U}\left(\mathbf{w}_{R}^{*}\right)$. We have by definition

$$
\lambda_{R} \mathcal{U}\left(\mathbf{w}_{R}^{*}\right)-\lambda_{L} \mathcal{U}\left(\mathbf{w}_{R}^{*}\right)=\lambda_{R}\left(\frac{\left(q^{*}\right)^{2}}{2 h_{R}^{*}}+g \frac{\left(h_{R}^{*}\right)^{2}}{2}\right)-\lambda_{L}\left(\frac{\left(q^{*}\right)^{2}}{2 h_{L}^{*}}+g \frac{\left(h_{L}^{*}\right)^{2}}{2}\right) .
$$

Since we clearly have by $(3.24)$

$$
\left\{\begin{aligned}
\left(q^{*}\right)^{2} & =\left(q_{0}^{*}\right)^{2}+2 q_{0}^{*} q_{1}^{*} \Delta z+\left(q_{1}^{*}\right)^{2}(\Delta z)^{2} \\
& =\left(q^{H L L}\right)^{2}+2 q^{H L L} q_{1}^{*} \Delta z+\left(q_{1}^{*}\right)^{2}(\Delta z)^{2}+O\left(\Delta x^{3}\right) \\
\left(h_{L}^{*}\right)^{2} & =\left(h^{H L L}\right)^{2}+2 h^{H L L} h_{1}^{*} \Delta z+\left(h_{1}^{*}\right)^{2}(\Delta z)^{2}+\Delta z \epsilon(\Delta z) \\
\left(h_{R}^{*}\right)^{2} & =\left(h^{H L L}\right)^{2}+2 h^{H L L}\left(\frac{\lambda_{L}}{\lambda_{R}}\right) h_{1}^{*} \Delta z+\left(h_{1}^{*}\right)^{2}\left(\frac{\lambda_{L}}{\lambda_{R}}\right)^{2}(\Delta z)^{2}+\Delta z \epsilon(\Delta z)
\end{aligned}\right.
$$

it comes

$$
\lambda_{R} g \frac{\left(h_{R}^{*}\right)^{2}}{2}-\lambda_{L} g \frac{\left(h_{L}^{*}\right)^{2}}{2}=\left(\lambda_{R}-\lambda_{L}\right) g \frac{\left(h^{H L L}\right)^{2}}{2}+\Delta z \epsilon(\Delta z)
$$

and

$$
\begin{aligned}
\frac{\left(q^{*}\right)^{2}}{2 h_{R}^{*}} & =\frac{\left(q^{*}\right)^{2}}{2 h^{H L L}}+\frac{\left(q^{*}\right)^{2}}{2\left(h^{H L L}\right)^{2}} \frac{1-\alpha}{\alpha} h_{1}^{*} \Delta z+\Delta z \epsilon(\Delta z) \\
& =\frac{\left(q^{H L L}\right)^{2}}{2 h^{H L L}}+\frac{\left(q^{H L L}\right)^{2}}{2\left(h^{H L L}\right)^{2}} \frac{1-\alpha}{\alpha} h_{1}^{*} \Delta z+\frac{2 q^{H L L} q_{1}^{*}}{2 h^{H L L}} \Delta z+\Delta z \epsilon(\Delta z)+O\left(\Delta x^{3}\right) .
\end{aligned}
$$

Similarly, we have

$$
\frac{\left(q^{*}\right)^{2}}{2 h_{L}^{*}}=\frac{\left(q^{H L L}\right)^{2}}{2 h^{H L L}}-\frac{\left(q^{H L L}\right)^{2}}{2\left(h^{H L L}\right)^{2}} h_{1}^{*} \Delta z+\frac{2 q^{H L L} q_{1}^{*}}{2 h^{H L L}} \Delta z+\Delta z \epsilon(\Delta z)+O\left(\Delta x^{3}\right) .
$$

Thus, since $(1-\alpha) \lambda_{R}=-\alpha \lambda_{L}$ we get

$$
\lambda_{R} \frac{\left(q^{*}\right)^{2}}{2 h_{R}^{*}}-\lambda_{L} \frac{\left(q^{*}\right)^{2}}{2 h_{L}^{*}}=\left(\lambda_{R}-\lambda_{L}\right) \frac{\left(q^{H L L}\right)^{2}}{2 h^{H L L}}-g \frac{q^{H L L}}{h^{H L L}} \frac{h_{L} h_{R}}{\bar{h}} \Delta z+\Delta z \epsilon(\Delta z)+O\left(\Delta x^{3}\right) .
$$

With clear definition for $\mathcal{U}\left(\mathbf{w}^{H L L}\right)$, gathering together the previous formulas easily gives

$\lambda_{R} \mathcal{U}\left(\mathbf{w}_{R}^{*}\right)-\lambda_{L} \mathcal{U}\left(\mathbf{w}_{R}^{*}\right)=\left(\lambda_{R}-\lambda_{L}\right) \mathcal{U}\left(\mathbf{w}^{H L L}\right)-g \frac{q^{H L L}}{h^{H L L}} \frac{h_{L} h_{R}}{\bar{h}} \Delta z+\Delta z \epsilon(\Delta z)+O\left(\Delta x^{3}\right)$.

Since the HLL scheme [25] is well-known to satisfy, at least for sufficiently large values of $-\lambda_{L}$ and $\lambda_{R}$, the entropy inequality

$$
\mathcal{U}\left(\mathbf{w}^{H L L}\right) \leq \frac{\lambda_{R} \mathcal{U}\left(\mathbf{w}_{R}\right)-\lambda_{L} \mathcal{U}\left(\mathbf{w}_{L}\right)}{\lambda_{R}-\lambda_{L}}-\frac{\mathcal{F}\left(\mathbf{w}_{R}\right)-\mathcal{F}\left(\mathbf{w}_{L}\right)}{\lambda_{R}-\lambda_{L}}
$$

we immediately get the following asymptotic behaviour for $\Delta \mathcal{U}$ :

$$
\Delta \mathcal{U} \geq \mathcal{F}\left(\mathbf{w}_{R}\right)-\mathcal{F}\left(\mathbf{w}_{L}\right)+g \frac{q^{H L L}}{h^{H L L}} \frac{h_{L} h_{R}}{\bar{h}} \Delta z+\Delta z \epsilon(\Delta z)+O\left(\Delta x^{3}\right) .
$$


Since we deal with a smooth topography such that $\Delta z=O(\Delta x)$, we get

$$
\Delta \mathcal{U} \geq \mathcal{F}\left(\mathbf{w}_{R}\right)-\mathcal{F}\left(\mathbf{w}_{L}\right)+g \frac{q^{H L L}}{h^{H L L}} \frac{h_{L} h_{R}}{\bar{h}} \Delta z+\Delta x \epsilon(\Delta x)
$$

and to conclude it suffices to set

$$
\sigma_{\nu}\left(\Delta t, \Delta x ; \mathbf{v}_{L}, \mathbf{v}_{R}\right)=g \frac{q^{H L L}}{h^{H L L}} \frac{h_{L} h_{R}}{\bar{h}} \frac{\Delta z}{\Delta x}
$$

which is clearly consistent with $g h u \partial_{x} z . \mathbf{\square}$

6. Conclusions. We have considered in this paper the design of two new numerical schemes based on suitable approximate Riemann solvers for the shallow-water equations. A particular attention has been paid to the more difficult aspects of wellbalancing of moving water equilibria, guaranteeing non-negative water heights, and satisfying a discrete entropy inequality. These properties are not trivial. The first scheme is linear but the entropy inequality property is not proved, while the second one is non linear but satisfies the latter three aspects together. Up to our knowledge, existence of such a scheme was not clear and it was the purpose of this contribution to answer this question. We hope that it could open further research in that direction. The next step, which will be addressed in a forthcoming contribution, will be to see whether such schemes can be competitive and provide good or even better numerical results than existing schemes for general initial and boundary value problems. Promising preliminary results have been obtained but let us underline that a couple of key points have to be considered with care in order to implement the proposed schemes. More precisely, it is first proved that the eigenvalues $\lambda_{L}$ and $\lambda_{R}$ can be chosen such that the properties of the schemes are satisfied but it is not clear how to define them practically since asymptotic arguments have been used. This point is also important to define the time step $\Delta t$. Second, it is also a matter of evaluating the $C$ constant which denotes some $L^{\infty}$-bound of the $x$-derivative of $h$ in the region where $h$ admits a $x$-derivative.

The proposed schemes are first-order accurate. It would be interesting to study the second-order extensions in both space and time, while still preserving the validity of the three properties. For the time being, we are not able to prove the discrete entropy inequalities in this context.

The proposed schemes are also 1D. Extensions to several space dimensions are always possible by considering convex combinations of the proposed 1D schemes, in the spirit of [4], [5] (see also [36]). The 2D extensions are expected to inherit the positivity and entropy properties of the 1D schemes. Regarding the well-balanced property, the lake at rest will be preserved but the moving steady states are not expected to be exactly preserved in general because of the mesh rotations.

\section{REFERENCES}

[1] E. Audusse, F. Bouchut, M. O. Bristeau, R. Klein, B. Perthame, A fast and stable well-balanced scheme with hydrostatic reconstruction for shallow water flows, SIAM J.Sci.Comp., 25, 2050-2065 (2004).

[2] A. Bermudez, M.E. Vazquez-Cendon, Upwind Methods for Hyperbolic Conservation Laws with Source Terms, Computers and Fluids. 23 1049-1071 (1994).

[3] C. Berthon, Numerical approximations of the 10-moment Gaussian closure, Math. Comput., $75,1809-1831$ (2006). 
[4] C. Berthon, Robustness of MUSCL schemes for 2D unstructured meshes, J. Comp. Physics 218 (2006) 495-509.

[5] C. Berthon, F. Foucher, Efficient well-balanced hydrostatic upwind schemes for shallowwater equations, J. Comput. Phys., 231 (2012) pp. 4993-5015

[6] F. Bouchut, Non-linear stability of finite volume methods for hyperbolic conservation laws and well-balanced schemes for sources, Frontiers in Mathematics, Birkhauser, 2004.

[7] F. Bouchut, C. Klingenberg, K. Waagan, A multiwave approximate Riemann solver for ideal MHD based on relaxation. I. Theoretical framework, Numer. Math., 108, 7-42 (2007).

[8] F. Bouchut, C. Klingenberg, K. Waagan, A multiwave approximate Riemann solver for ideal MHD based on relaxation II: numerical implementation with 3 and 5 waves, Numer. Math., 115, 647-0679 (2010)..

[9] F. Bouchut, T. Morales, A subsonic-well-balanced reconstruction scheme for shallow water flows, Siam J. Numer. Anal., 48(5):1733-1758, 2010.

[10] S. Bryson, Y. Epshteyn, A. Kurganov, G. Petrova, Well-balanced positivity preserving centralupwind scheme on triangular grids for the Saint-Venant system, ESAIM Math. Model. Numer. Anal., 45 (2011), 423-446.

[11] T. Buffard, T. Gallouët, J.M. Hérard, A naive Godunov scheme to solve shallow water equations, CR Acad. Sci. Paris, 326, 385-390 (1998).

[12] M.J. Castro, J.M. Gallardo, J.A. López-García, C. Parés, Well-balanced high order extensions of Godunov's method for semilinear balance laws, SIAM J. Num. Anal., 46 (2008), 1012 1039.

[13] M. J. Castro, A. Pardo, C. Parés, Well-balanced numerical schemes based on a generalized hydrostatic reconstruction technique, Mathematical Models and Methods in Applied Sciences, 17 (2007), 2065-2113.

[14] C. Chalons, F. Coquel, E. Godlewski, P-A Raviart, N. Seguin. Godunov-type schemes for hyperbolic systems with parameter dependent source. The case of Euler system with friction. Math. Models Methods Appl. Sci., (2010).

[15] C. Chalons, J.-F. Coulombel, Relaxation approximation of the Euler equations, J. Math. Anal. Appl., 348 (2), 872-893 (2008).

[16] A. Chinnayya, A.-Y. LeRoux, N. Seguin, A well-balanced numerical scheme for the approximation of the shallow-water equations with topography: the resonance phenomenon, International Journal on Finite Volume (electronic), volume 1(1), 1-33 (2004).

[17] G. Gallice. Solveurs simples positifs et entropiques pour les systèmes hyperboliques avec terme source. C. R. Math. Acad. Sci. Paris 334, no. 8, 713-716 (2002).

[18] G. Gallice. Positive and entropy stable Godunov-type schemes for gas dynamics and MHD equations in Lagrangian or Eulerian coordinates. Numer. Math. 94 no.4, 673-713 (2003).

[19] T. Gallouët, J.M. Hérard, N. Seguin, Some approximate Godunov schemes to compute shallowwater equations with topography, Computers and Fluids, 32, 479-513 (2003).

[20] E. Godlewski and P.-A. Raviart. Numerical approximation of hyperbolic systems of conservation laws. Applied Mathematical Sciences, vol. 118, Springer-Verlag, New York, (1996).

[21] L. Gosse, A well-balanced flux-vector splitting scheme designed for hyperbolic systems of conservation laws with source terms, Comput. Math. Appl. 39 (2000), 135-159.

[22] N. Goutal, F. Maurel, A finite volume solver for 1D shallow-water equations applied to an actual river, Int. J. Numer. Meth. Fluids 38, 1-19 (2002).

[23] N. Goutal, F. Maurel, Proceedings of the 2nd workshop on dam-break wave simulation, Technical report, EDF-DER, HE-43/97/016/B (1997)

[24] J. M. Greenberg, A. Y. Leroux, A well-balanced scheme for the numerical processing of source terms in hyperbolic equations, SIAM J. Numer. Anal., 33, 1-16 (1996).

[25] A. Harten, P.D. Lax, B. Van Leer, On upstream differencing and Godunov-type schemes for hyperbolic conservation laws, SIAM Review, 25, 35-61 (1983).

[26] S. Jin, A steady-state capturing method for hyperbolic systems with geometrical source terms, M2AN Math. Model. Numer. Anal., 35, 631-645 (2001).

[27] P.D. Lax, Hyperbolic systems of conservation laws, II CPAM, 10 (1957), 537-566

[28] P.G. LeFloch, M.D. Thanh, A Godunov-type method for the shallow water equations with discontinuous topography in the resonant regime, Journal of Computational Physics, 230 (2011), 7631-7660.

[29] P.G. LeFloch, M.D. Thanh, The Riemann problem for shallow water equations with discontinuous topography, Com. Math. Sci., 5(4), (2007), 865-885.

[30] Q. Liang, F. Marche, Numerical resolution of well-balanced shallow water equations with complex source terms, Advances in Water Resources, 32 (6), 873-884 (2009).

[31] T. Morales de Luna, M. J. Castró Diaz, C. Parés, E. Fernández Nieto, On a shallow water model for the simulation of turbidity currents, Commun. Comput. Phys. 6 (2009), 848-882. 
[32] S. Noelle, N. Pankratz, G. Puppo, J.R. Natvig, Well-balanced finite volume schemes of arbitrary order of accuracy for shallow water flows, J. Comp. Phys., 213, 474-499 (2006).

[33] S. Noelle, Y. Xing, C.W. Shu, High order well-balanced finite volume WENO schemes for shallow water equations with moving water, J. Comput. Phys., 226 (1), 29-58 (2007).

[34] C. Parés, M. Castro, On the well-balance property of Roes method for nonconservative hyperbolic systems. Applications to shallow-water systems, Mathematical Modelling and $\mathrm{Nu}-$ merical Analysis., 38 (2004), 821-852.

[35] P.-A. Raviart, L. Sainsaulieu, A nonconservative hyperbolic system modeling spray dynamics. Part 1. Solution of the Riemann problem, Mathematical Models and Methods in Applied Sciences, 5(3), 297-333 (1995).

[36] B. Perthame, Y. Qiu, A variant of Van Leers method for multidimensional systems of conservation laws, J. Comput. Phys. 112 (1994) 370381.

[37] V. V. Rusanov, The calculation of the interaction of non-stationary shock waves with barriers. (Russian). Zh. Vychisl. Mat. Mat. Fiz., 1961, Volume 1, Number 2, 267-279

[38] G. Russo, A. Khe, High order well-balanced schemes based on numerical reconstruction of the equilibrium variables, Proceedings "WASCOM 2009" 15th Conference on Waves and Stability in Continuous Media, 230241, World Sci. Publ., Hackensack, NJ, 2010.

[39] G. Russo, A. Khe, High order well balanced schemes for systems of balance laws, Hyperbolic problems: theory, numerics and applications, 919-928, Proc. Sympos. Appl. Math., 67, Part 2, Amer. Math. Soc., Providence, RI, 2009.

[40] Y. Xing, Exactly well-balanced discontinuous Galerkin methods for the shallow water equations with moving water equilibrium, J. Comput. Phys. 257 (2014) 536-553.

[41] Y. Xing, C.-W. Shu, High order well-balanced finite volume WENO schemes and discontinuous Galerkin methods for a class of hyperbolic systems with source terms, J. Comput. Phys. 214 (2006) 567-598.

[42] Y. Xing, C.-W. Shu, S. Noelle, On the advantage of well-balanced schemes for moving-water equilibria of the shallow water equations, J. Sci. Comput. 48 (2011) 339-349.

[43] Y. Xing, X. Zhang, C.-W. Shu, Positivity-preserving high order well-balanced discontinuous Galerkin methods for the shallow water equations, Adv. Water Resour. 33 (2010) 14761493. 